\title{
RHEOMETRY OF DENSE GRANULAR COLLAPSE ON INCLINED PLANES
}

\author{
A Thesis \\ Submitted To the Faculty of Graduate Studies and Research \\ In Partial Fulfillment of the Requirements \\ For the Degree of \\ Masters of Applied Science \\ in \\ Environmental Systems Engineering \\ University of Regina \\ By \\ Olalekan Abdul-Azeez Rufai \\ Regina, Saskatchewan \\ July, 2018 \\ (C) Copyright 2018: Olalekan A. Rufai
}




\section{UNIVERSITY OF REGINA}

\section{FACULTY OF GRADUATE STUDIES AND RESEARCH SUPERVISORY AND EXAMINING COMMITTEE}

Olalekan Abdul-Azeez Rufai, candidate for the degree of Master of Applied Science in Environmental Systems Engineering, has presented a thesis titled, Theometry of Dense Granular Collapse on Inclined Planes, in an oral examination held on July 23, 2018. The following committee members have found the thesis acceptable in form and content, and that the candidate demonstrated satisfactory knowledge of the subject material.

External Examiner: $\quad$ Dr. Wei Peng, Industrial Systems Engineering

Supervisor: $\quad$ Dr. Yee-Chung Jin, Environmental Systems Engineering

Committee Member: $\quad$ Dr. Tsun Wai Kelvin Ng, Environmental Systems Engineering

Committee Member: $\quad$ Dr. Peng Wu, Environmental Systems Engineering

Chair of Defense: $\quad$ Dr. Kenneth Leyton-Brown, Department of History 


\begin{abstract}
The continuum deformation of gravity-driven dense granular materials on steep inclined planes exhibit similar flow phenomenon to natural and industrial occurrences. This kind of granular flow dynamics corresponds to unsteady flows which currently lack any theoretical formulations for its kinematic properties.

The implementation of the $\mu(I)$ rheology model and the Moving Particle Semiimplicit (MPS) mesh-free method as a coupled set is used to predict quantitatively the flow properties and phenomena of the granular materials on inclined planes. During deformation of the granular materials, transition of flow regime shows that the velocity profile is maintained at the dense region but fluctuates when tending to the dilute region due to limited interaction between granular materials.
\end{abstract}

The flow pattern of the granular materials shows the development of multiple shear band behaviour resulting from the influence of gravity on the flowing granular materials depending on different Froude numbers. The granular materials shear stress is found to attain a yield point before reducing as the shear rate increases exhibiting a thixotropic behaviour.

We also compared the velocity profile, shear-band, surface profile and wave front of the flowing granular materials with physical studies on a two-dimensional configuration giving acceptable qualitative agreements. 


\section{ACKNOWLEDGEMENTS}

The work presented in this thesis would not have been possible without the support of many dedicated people. I am particularly indebted to my master's supervisor Dr. Yee-Chung Jin for his mentorship, patience and support throughout all stages of this study. He is one of the distinguished human I ever got to know.

I would also like to thank the members of my thesis committee for their effort and time in reviewing my thesis with their valuable comments and suggestions.

I would like to thank Dr. Yih-Chin Tai and his graduate research students for their support and assistance in providing experimental data for my thesis.

I acknowledge the financial support of the Faculty of Graduate Studies and Research, at the University of Regina and the Natural Science and Engineering Research Council of Canada.

Finally, I would like to thank my parents, siblings, fiancée, family members and friends for their great support and encouragement. Thank you all for always being there for me. 


\section{TABLE OF CONTENTS}

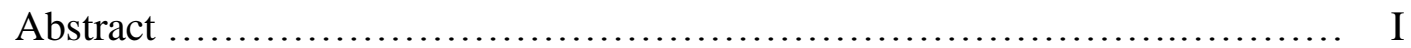

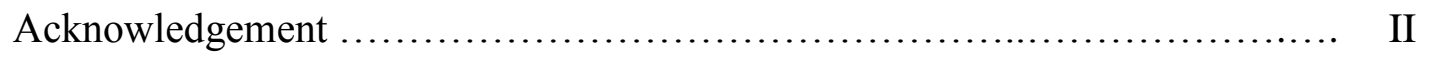

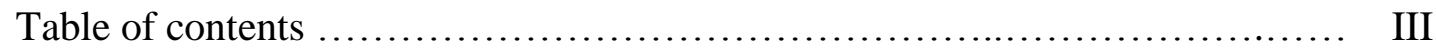

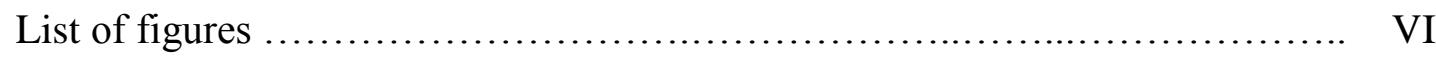

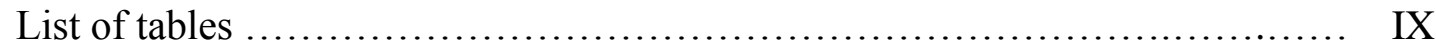

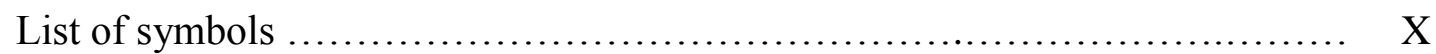

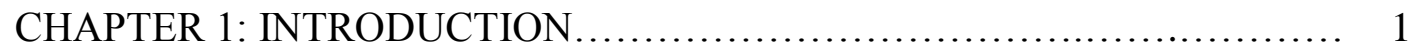

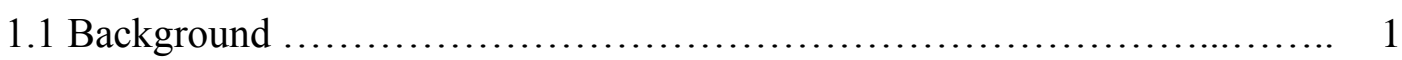

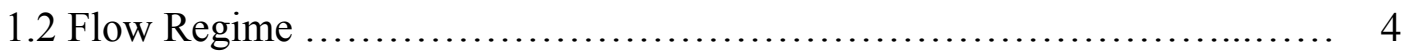

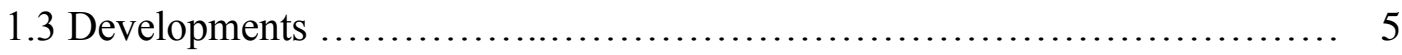

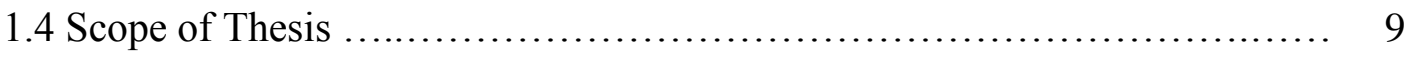

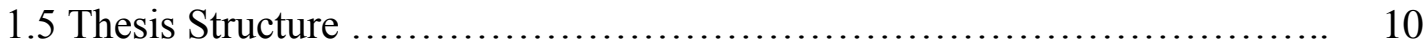

CHAPTER 2: EXPERIMENTAL SET UP AND THEORETICAL FORMULATION

2.1 Experimental setup ............................................. 11

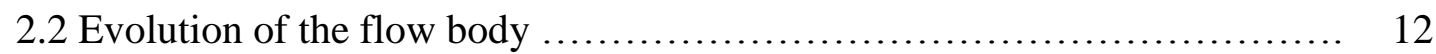

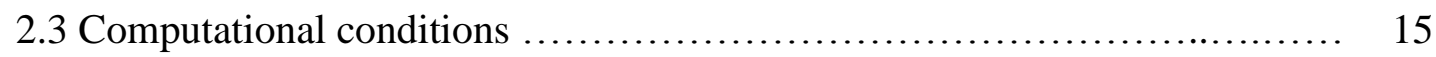




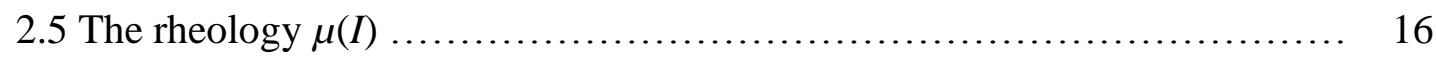

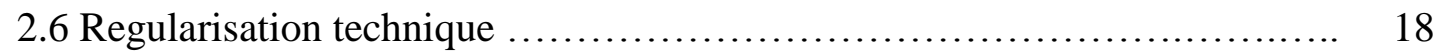

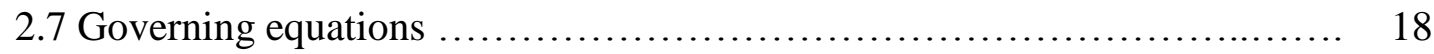

CHAPTER 3: NUMERICAL METHOD ................................. 20

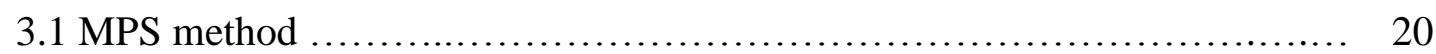

3.1.2 Requirement of the Kernel Function ........................... 22

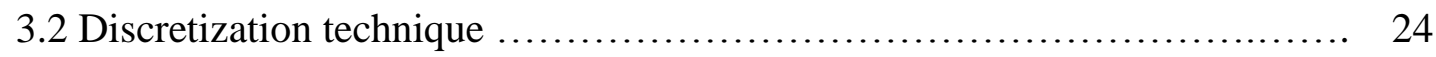

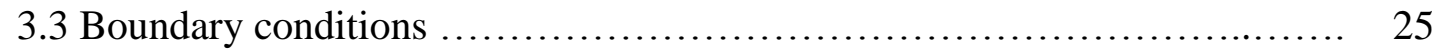

CHAPTER 4: RESULT AND DISCUSSION ........................... 27

4.1 Properties of the granular flow behaviour ............................. 27

4.2 Velocity profile ................................................ 27

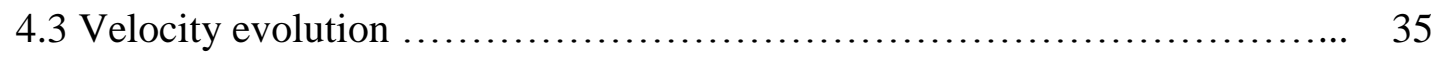

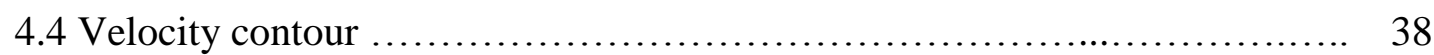

4.5 Shear banding in unsteady states ................................. 41

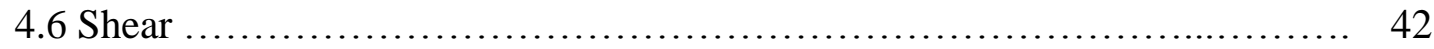

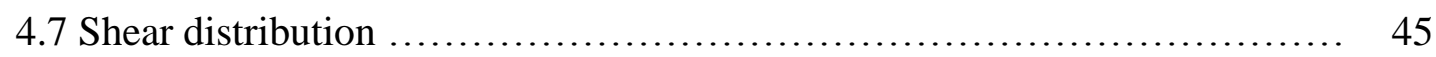

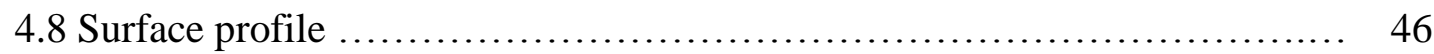




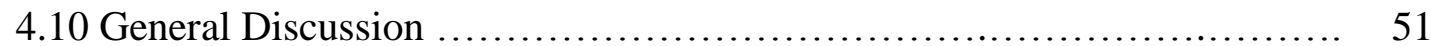

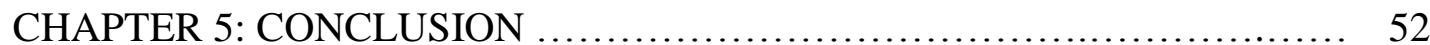

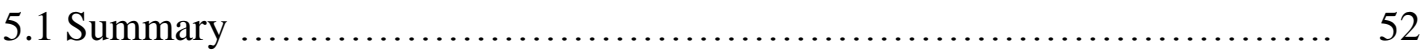

References ............................................................ 55 


\section{LIST OF FIGURES}

Fig. 1.1: Examples of industrial and geophysical granular processes

Fig. 1.2: Typical granular materials

Fig. 2.1: Experimental setup

Fig.2.2: The series of snapshots for an experiment with glass bead granules for $45^{\circ}$ slope

Fig. 2.3: The series of snapshots for an experiment with glass bead granules for $30^{\circ}$ slope 14

Fig. 3.1: Plotted kernel functions from table 3.1

Fig. 4.1 Horizontal velocity profile for $45^{\circ}$ slope showing comparison between experimental and numerical results at a time $t(\mathrm{~s})$ from specific locations

Fig. 4.2 Vertical velocity profile for $45^{\circ}$ slope showing comparison between experimental and numerical results at a time $t(\mathrm{~s})$ from specific locations 30

Fig. 4.3 Horizontal velocity profile for $30^{\circ}$ slope showing comparison between experimental and numerical results at a time $t(\mathrm{~s})$ from specific locations

Fig. 4.4 Vertical velocity profile for $30^{\circ}$ slope showing comparison between experimental and numerical results at a time $t(\mathrm{~s})$ from specific locations 
Fig. 4.5 Velocity evolution during acceleration down a slope of $45^{\circ}$ during initialisation when the granular materials start to deform at fixed sections

Fig. 4.6 Velocity evolution during acceleration down a slope of $30^{\circ}$ during initialisation when the granular materials start to deform at fixed sections

Fig. 4.7 Velocity contour plot profile for $45^{\circ}$ slope showing comparison between experimental and numerical results for time (a) $0.05 \mathrm{~s} \mathrm{(b)} 0.10 \mathrm{~s} \mathrm{(c)} 0.15 \mathrm{~s} \mathrm{(d)} 0.2 \mathrm{~s}$ (e) $0.4 \mathrm{~s}$ 39

Fig. 4.8 Velocity contour plot profile for $30^{\circ}$ slope showing comparison between experimental and numerical results for time (a) $0.05 \mathrm{~s}$ (b) $0.10 \mathrm{~s}$ (c) $0.15 \mathrm{~s} \mathrm{(d)} 0.2 \mathrm{~s}$ (e) $0.4 \mathrm{~s}$ 40

Fig. 4.9 Multi-Shear band layers for $45^{\circ}$ slope showing comparison between experimental and numerical results for time (a) $0.05 \mathrm{~s}$ (b) $0.10 \mathrm{~s}$ (c) $0.15 \mathrm{~s} \mathrm{(d)} 0.20 \mathrm{~s}$ (e) $0.30 \mathrm{~s}$

Fig. 4.10 Multi-Shear band layers for $30.8^{\circ}$ slope showing comparison between experimental and numerical results for time (a) $0.05 \mathrm{~s}$ (b) $0.10 \mathrm{~s}$ (c) $0.15 \mathrm{~s}$ (d) $0.20 \mathrm{~s}$ (e) $0.30 \mathrm{~s}$ 44

Fig. 4.11 Rheological behaviour of the granular materials where (a) and (b) shows shear stress and shear rate as a function of time and (c) to (d) shows the friction coefficient and shear stress as a function of shear rate

Fig. 4.12 Shear stress and shear rate for $45^{\circ}$ slope 47 
Fig. 4.13: Surface profile for $45^{\circ}$ slope showing comparison between experimental and numerical results for time (a) $0.05 \mathrm{~s} \mathrm{(b)} 0.10 \mathrm{~s}$ (c) $0.15 \mathrm{~s}$ (d) $0.2 \mathrm{~s}$

Fig. 4.14 Surface profile for $30^{\circ}$ slope showing comparison between experimental and numerical results for time (a) $0.05 \mathrm{~s}$ (b) $0.10 \mathrm{~s} \mathrm{(c)} 0.15 \mathrm{~s}$ (d) $0.2 \mathrm{~s}$ 49

Fig. 4.15 Wave front time evolution for $45^{\circ}$ and $30^{\circ}$ slope showing comparison between experimental and numerical results 51 


\section{LIST OF TABLES}

Table 2.1 Geometry and simulation parameters

Table 3.1 List of other successful weighted functions 


\section{LIST OF SYMBOLS}

$I=$ inertia number

$\mu(I)=$ friction coefficient

$P=$ pressure

$d_{g}=$ grain size in diameter

$\rho_{s}=$ density of granular materials

$\tau_{i}=$ local tangential shear stress

$\gamma_{i j}=$ strain rate tensor

$\gamma=$ second invariant

$\partial_{j} u_{i}=$ partial derivatives of velocity components in the $i$ direction

$\partial_{i} u_{j}=$ partial derivatives of velocity components in the $j$ direction

$\theta_{s}=$ static frictional angles

$\theta_{2}=$ dynamic frictional angles

$\sigma_{i j}=$ Cauchy stress tensor

$\varepsilon=$ volume of fraction

$\delta_{i j}=$ unit tensor

$\tau_{i j}=$ deviatoric stress

$I_{0}=$ empirical coefficient constant

$\mu_{s}=$ static friction coefficient 
$\mu_{2}=$ dynamic friction coefficient

$\eta_{R}=$ regularisation technique

$\alpha_{r}=$ regularisation convergence value

$\mathbf{u}=$ particle velocity vector

$t=$ time

$g$ = gravity

$\rho=$ density for fluid

$\rho_{s}=$ granular materials density

$\mu=$ dynamic viscosity

$\tau=$ stress tensor

$\omega(r)=$ the kernel function

$r_{e}=$ radius of the interaction circle around each targeted particle

$d_{l}=$ particle size

$r=$ distance between a target particle $i$ and one of its neighboring particle $j$

$\langle n\rangle_{i}=$ particle number density

$\langle\rho\rangle_{i}=$ real fluid density

$m_{i}=$ is the mass of the fluid particle

$\phi=$ arbitrary scalar term

$n^{0}=$ initial particle density 
$n_{i}=$ under the incompressible flow

$\lambda=$ coefficient used to scale the variance for consistency

$\beta=$ threshold coefficient

$\zeta=$ coefficient used in the equation of state

$c_{0}=$ is the artificial sound speed in the reference medium

$\theta_{r}=$ angle of repose

$(h)=$ depth of the granular flow

$\stackrel{x}{u}=$ dimensionless velocity

$\stackrel{x}{y}=$ dimensionless position of granular material

$v_{y}=$ vertical velocity component

$v_{x}=$ horizontal velocity component

$\left.\langle\nabla \phi\rangle\right|_{i j}=$ gradient operator

$\left.\left\langle\nabla^{2} \phi\right\rangle\right|_{i j}=$ Laplacian operator 


\section{CHAPTER 1: INTRODUCTION}

\subsection{Background}

The study of physical science shows to human existence how important it is to understand matter and energy. The complexities in the phase classification of matter which is unique in dense granular flows result in the need to further understand the rheological properties of the particulate system during geophysical transition and in formulating a general constitutive relationship (Berzi et al. 2011; Jop et al. 2005; Jop, 2015; Kumaran, 2006; MiDi, 2004; Pouliquen et al. 2009; Shaninpoor and Siah, 1981; Shahinpoor, 1983). Natural hazardous events exhibit complex rheologies which are associated with geophysical granular flows like landslides, debris flows, avalanches and pyroclastic flows (Forterre and Pouliquen 2008; Pouliquen and Forterre 2002). However, having a broad knowledge in this area would be significant for both scientific accomplishment and engineering application to mitigate risk from such hazards. Previous studies has been completed to gain a better understanding of the behaviour of dense granular flows on different flow configurations namely plane shear, coquette cell, vertical silo, inclined plane, heap flow and rotating drum (Ancey, 2001; Azanza et al. 1999; Bagnold, 1954; Cui and Gray, 2013; Da Cruz et al. 2005; Dunatunga et al. 2015; Forterre and Pouliquen, 2008; MiDi, 2004; Pouliquen and Forterre 2009; Shukla and Meheboob, 2013; Xu and Jin, 2016). Despite the study of these various configurations, the inclined plane represents a close relationship to real world situations as the collisions and frictional contacts exhibit complex properties during particulate flows. These kind of dry particulate flows phenomenon are centered on the effect of instantaneous application of gravitational force or other external forces and the internal friction force between granular materials. 
The interaction between granular particles can be classified to exhibit plasticity behaviour during deformation. The kinetic theories which are dependent on a uniform arrest or infinite flow play a significant role in the description of the granular flow. Depending on the thickness of the flowing materials, the collisional flows are characterised with a certain local order depending on the density profile and distribution which are dense near the bed and very dilute at the free surface (Azanza et al. 1999). Several geophysical flows occur both in the industry and nature as shown in Fig. 1.1. In most cases the flows are destructive leading to loss of life and properties. Granular materials are found all around us in various forms, few examples of granular materials are shown in Fig. 1.2. The sensitivity of the granular flow varies for different configuration and parameters. Some of these parameters are the channel inclination, shape of the grains, granulometry, coupling with the interstitial fluid and to a lesser extent the flow rate and the kind of roughness (Jop et al. 2006; Azanza et al. 1999; Ancey, 2002; Pouliquen and Chevoir, 2002; Goujon, 2003). Depending on the configuration and sensitivity of parameters, deformation of the granular materials during shear may be classified as compact when the material becomes loose or dilatant when dense (Pouliquen et al. 2002) resulting into the development and transition of flow regimes. 

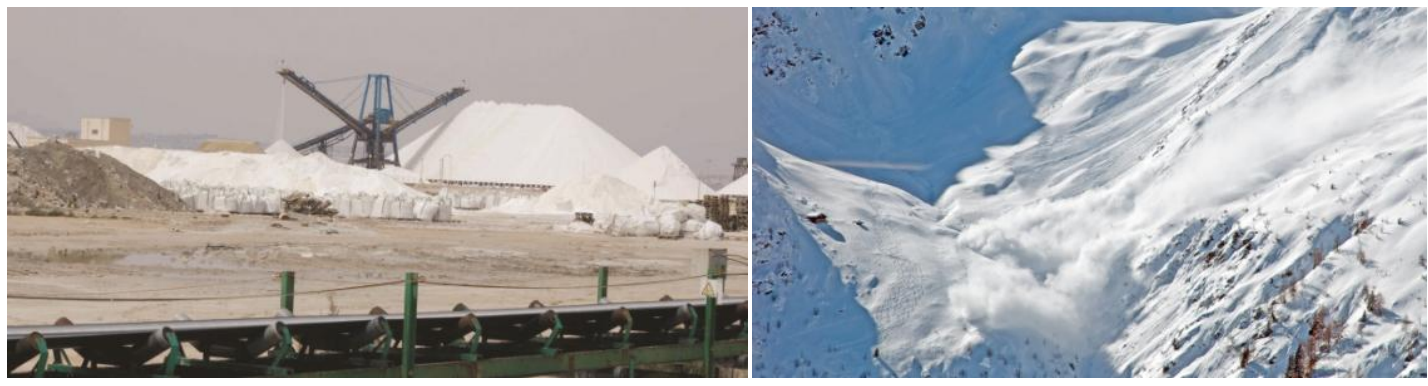

Fig. 1.1: Examples of industrial and geophysical granular processes ${ }^{1}$. This is a public domain photo from http://nsem.info/know-risks/avalanche

(a)

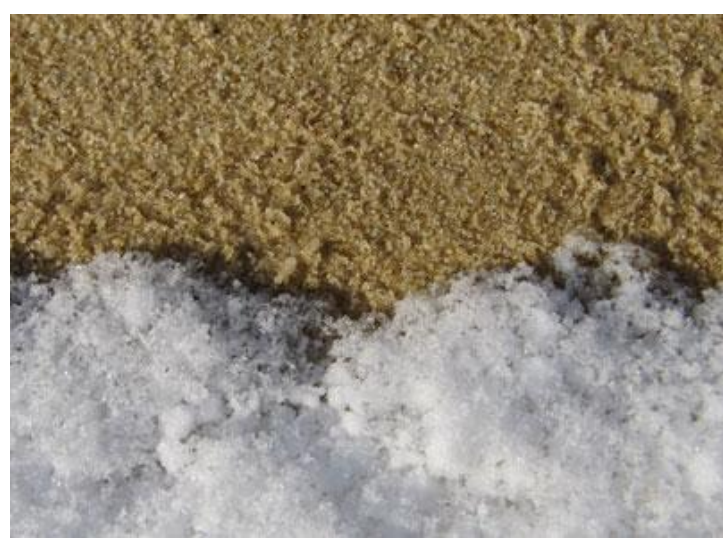

(b)

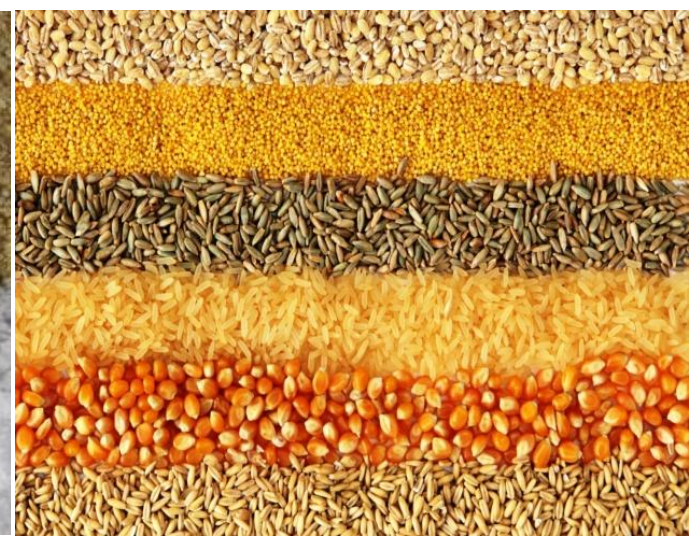

Fig. 1.2: Typical granular materials ${ }^{1}$.

a. This is a public domain photo from http://www.realfoodforlife.com/11-gluten-freegrains/

b. This is a public domain photo from http://adnams.co.uk/about/news/hotelnews/nature-environment/southwold-snow-in-pictures/ 


\subsection{Flow Regime}

The transfer of momentum and dissipation of energy from inter-particle collision leads to the formulation of a rheology from kinetic theories, where the resulting regime is termed as a collision or kinetic regime which is as a function of the inclination range and particle flux (Azanza et al. 1999). The inclined flow dynamics is related to two basic angles which are the angle of repose and maximum angle of stability, both known as the material properties (Azanza et al. 1999; Silbert et al. 2003). The former is dependent on gravity and empirically observed from experimental or numerical studies which are the static state of the granular materials while the latter in most cases if greater than the angle of repose induces flow. This induced flow would stop only when force balance occur between gravity and the friction generated by energy transfer and dissipation between granular materials during multi-collision, otherwise the materials flow continuously until it approaches the end of the channel or when obstructed (Ancey, 2001; Pouliquen and Chevoir, 2002). A flow to no flow or continuous flow is determined from the relativity of these angles which could result to a steady state flow, unsteady state flow or no flow (Ancey, 2001; Azanza et al. 1999; Pouliquen and Chevoir, 2002). A static or flow at rest when the inclination angle exceeds the angle of repose of the flowing granular materials termed as a steady uniform or continuous flow when the inclination angle exceeds the maximum angle of stability becoming rapidly unstable and producing a stationary wedge close to the boundary (Ancey, 2001). Since unstable flow is mostly determined by high flow rates or inclination, the free surface mostly experiences deformation in the transverse section and sometime may lead to longitudinal vortices as observed by Forterre et al. (2001) and Shukla and Meheboob, (2013). 
The rate of transition between the solid and liquid phase is very short and done in substrate layers with uniform velocities until a minimum depth is reached. The dense flow regime is characterised as an inelastic collision where several interactions occurs between the particles which is mostly determined from the degree of slope and the angle of repose of the granular material. Inclined flows may behave as solid, liquid or gas (Ancey, 2002; Pouliquen et al. 2002). Under mild inclination or effect of microgravity during initialisation, the flow of granular material is termed a solid and a liquid for steeper inclinations. A solid flow regime is mostly referred to as the quasi-static as described from the plasticity characteristics where deformation of the material yields along slip plane as a result the ratio of tangential to normal stress to the internal friction of the granular material (Pouliquen and Chevoir, 2002).

\subsection{Developments}

Early mathematical formulations have been empirical and suggested by Bagnold (1954) since the mid 90's to be related to the mechanism found in the exchange of momentum between different particles collision occurring simultaneously for a specific time or infinitely depending on the flow type (Bagnold, 1954). Although this theory serves as the basis for several studies over the years, most recent studies show different flow properties while maintaining a similar configuration which might be linked to different experimental setup, sensitivity to the roughness conditions and flow instabilities in the form of vortices (Forterre and Pouliquen, 2001; Lo et al. 2010).

A complexity from the behaviour of the granular materials makes it easy to deal with arresting flows compared to those which flow continuously. The flow dynamics and characteristics at certain time for the continuous flow during 
deformation are yet to be established. This brings about the friction between boundary layer and the use of two-dimensional geometry which would not capture the 3-d effect of the flow (Azanza et al. 1999). Rheology model sometimes don't capture dilute particles (Xu and Jin, 2016), and in some cases require fitting of parameters with the use of dimensionless variables (Chauchat and Médale, 2014). The formulation of varieties of model exists but with few contradictions, yet giving acceptable agreement with experimental studies for some flow phenomenon and profile characteristics (Pouliquen and Chevoir, 2002).

Shaninpoor and Siah (1981) and Shaninpoor (1983) further developed on Bagnold formulation for rapid flow using inter-granular collisions, friction and boundary conditions to describe the shear and normal stresses in the flow while comparing it with experimental observations. The given expression tends to classical results at a zero friction. It is expected that increase in inclination would present more complexities in the flow regime as instabilities generated leads to spontaneous pattern formulation resulting to longitudinal or spanwise vortices (Börzsönyi, 2009; Brodu et al. 2015; Forterre and Pouliquen, 2001). Since the instability of such flows makes it difficult to study, Brodu et al. (2015) introduces a frictional sidewall to initiate the addition of particle to wall frictional forces in order to balance the driving force using Discrete Element Method (DEM) method for simulating the flow. Coefficient of restitution and frictional coefficient was used to control the inelastic collision between particles and wall collision as the contact force using periodical boundary conditions. The investigation focused on flows on inclination greater than $30^{\circ}$ generating a support flow regime with stabilised dense core within flustered regions close to the wall producing instabilities. This dense core exhibit constant properties over time and are located above highly agitated granular gaseous regime. At higher 
inclination, the core transits to granular gas with an approximate constant depth. Overall a scaling law from the exploration of the angle and mass holdup was used to describe the velocity using a power law of $q \alpha h^{1.25}$.

The higher the degree of inclination, the more difficulty it takes the flow in reaching a steady state. Since the frictional angle is material dependent, shallow slopes greater than the angle of repose are also classified as continuous flow which may attain a steady state. Stanislav et al. (2016) and Jessop et al. (2017) derived theoretical prediction for velocity, shear stress and shear rate for a constant thickness of the granular material on shallow inclined slope $\left(17^{\circ}\right.$ to $\left.25^{\circ}\right)$. Although the frictional interaction is classified to be unsteady, energy from the collision of particles balances with the dissipation leading to a steady state and finally an arrest flow. The propositions was based on the gravitational and frictional forces acting as resistivity and driving force with rheological measurements sensitive to the flow characteristics while friction increases with shear rate under constant normal stress and linearly with the inertia number. The analytical model was validated using Discrete Element Method (DEM) numerical simulation method. Jessop et al. (2017) also proposed a mathematical formulation from a continuum description of two inter-penetrating phases interaction for unsteady developing flows on slopes for velocity, front wave and position while focusing on the transition from unsteady to uniform behaviour of fluidised current on shallow slopes.

Several studies related to dry granular flow on inclined planes were mostly completed by experimental and computer simulations. Experimental studies are limited in the literature due to difficulties in capturing rapid deformation of granular materials with constrained formulations. The behaviour of the granular materials during deformation have led to different propositions of its constitutive laws which 
are then extended to simple rheology models used in predicting granular flows. Lagrée et al (2011) conducted comparative studies on some of the rheology models such as the $\mu(I)$ rheology, Newtonian, Bingham, Bagnold and the constant friction for granular column collapse and found that the $\mu(I)$ rheology model is capable of predicting most of its deformation dynamics. The incorporation of the $\mu(I)$ rheology model in an incompressible two or three dimensional fluid equation have inspired many numerical simulation methods for granular flows (Barker et al. 2015 and 2017; Ionescu, 2015; Lagrée et al. 2011; Tankeo et al. 2013).

Most granular flow Simulations have mainly been carried out using a continuum or discrete modeling approach. One of the simulation method used is the particle method such as the Moving Particle Semi-implicit method (MPS) (Koshizuka and Oka 1996), Smoothed Particle Hydrodynamics (SPH) (Lucy, 1977; Gingold and Monaghan, 1977) and the Material Point method (MPM) (Sulsky et al. 1994). The Smoothed Particle Hydrodynamics (SPH) method have been used to simulate granular collapse on an inclined bed (Ikari and Gotoh, 2016), free-surface flows (Chambon et al. 2011), and granular column collapse (Szewc, 2017). The Material Point Method has also been employed by Dunatunga and Kamrin, (2015) and Fei et al. (2012) to study granular flows on inclined plane. The MPS method has been used to study granular flows on limited configurations like the granular column collapse (Xu et al. 2017) and dry and submerged granular flows (Jafari-Nodoushan et al. 2017). To further explore more configurations using this method, the MPS method is employed with the $\mu(I)$ rheology as a coupled set to simulate the granular flow on steep inclined planes. These granular flows are either steady or unsteady depending on the flow regime. The coupled set have been used for granular column collapse on horizontal planes and now extended to inclined planes in this current study. 
Previous studies were completed on steady granular flows leading to good understanding of its rheology, characteristics and proposition of empirical analytical methods compared to the unsteady state which is yet to be understood. Steady granular flows have been investigated using a range of inclination angles to show the flow kinematics (Fan et al. 2013; Ikari and Gotoh, 2016; Xu et al. 2017), run out distances (Ertas et al. 2001; Saingier et al. 2016), instabilities (Forterre and Pouliquen, 2003; Mollon, 2016; Shukla and Meheboob, 2013), deformation properties (Shojaaee et al. 2012; Tordesillas et al. 2016), scaling (Pouliquen, 1999; Pouliquen and Forterre, 2002) and rheology (Berzi et al. 2011; Jop et al. 2006; MiDi, 2004; Pouliquen and Forterre, 2009; Pouliquen et al. 2006). Although sensitive parameters relative to the flow are experimentally not accessible making it difficult to predict the rheological behaviours without making reasonable assumptions, numerical methods give room for further studies and understanding of the granular matter.

\subsection{Scope of Thesis}

The MPS method is coupled with the $\mu(I)$ rheology to simulate the granular flow on steep inclined planes. These granular flows are either steady or unsteady depending on the flow regime. The coupled methods have been used for granular column collapse on horizontal planes. This study will extend the granular collapse using the coupled MPS and $\mu(I)$ rheology model to inclined planes. Since only few studies have focused on unsteady granular flow, a simple description of unsteady granular flow in two dimensional configurations to predict the flow characteristics using both physical and numerical methods by giving detailed microscopic and macroscopic observations will be provided. Detail observation includes the velocity (profiles, evolution, and 
contours), surface profiles, wave front, shear, shear banding and distribution. The aim of this present study is to gain a greater understanding of the dynamics governing unsteady granular flows on inclined planes by incorporating the $\mu(I)$ inertial rheology model with the MPS particle method.

\subsection{Thesis Structure}

The thesis is organized according to the structure given below:

1. Chapter 2 gives a brief discussion on both experimental set up and results with the rheology model formulation for granular flows.

2. Chapter 3 describes the Moving particle semi-implicit method techniques and study of different available weighted function and trial on reviewing to state the most suitable in the MPS method for granular flows.

3. Chapter 4 presents both results and discussion for velocity profiles taking account of the horizontal and vertical components, velocity evolution, velocity contours, shear band, shear stress, and shear distribution, surface profile and wave fronts.

4. Chapter 5 gives a final remark on the conclusion of the work done. 


\section{CHAPTER 2: EXPERIMENTAL SET UP AND THEORETICAL FORMULATION}

\subsection{Experimental setup}

Experiments of quasi-2D were performed in a narrow channel, where glass beads were confined between glasses sheets with a net width of $20 \mathrm{~mm}$. Fig. 2.1 illustrates the experimental setup. The glass beads were initially held by a front gate, which is normal to the ground and top surface. The initial heap remains horizontal at the surface on $30.8^{\circ}$ and $45.2^{\circ}$ inclinations respectively. These angles are selected to achieve both stable and unstable flow while making it less difficult to extract physical measurements during experimental study. For all experiments, the total weight of the glass beads is maintained constant at $195 \mathrm{~g}$, where the diameter of the used glass beads is $2 \mathrm{~mm}$. For ease of PIV analysis, $20 \%$ of the glass beads are in black color while the remaining $80 \%$ are left plain. During the experimental runs, the front gate was lifted vertically, releasing the glass beads instantaneously (less than $0.04 \mathrm{~s}$ ) to similarly represent natural behaviour down inclined plane. The whole process of the collapse was captured by a high-speed CMOS digital camera (IDT inc., X-Stream III) at a speed of 1000 frames per second (fps). Velocity fields in the collapsing masses are obtained by applying the particle image velocimetry (PIV) technique (e.g. Lueptow et al. 2000), where an open-source package, PIVlab (e.g. Sarno et al. 2014; Thielicke and Tamhuis, 2014), is used.

The granular materials velocities are estimated using the particle image velocimetry (PIV) technique from snapshots taken by the CMOS digital camera. From these snapshots, surface profiles and wave front of the granular materials can also be determined. 


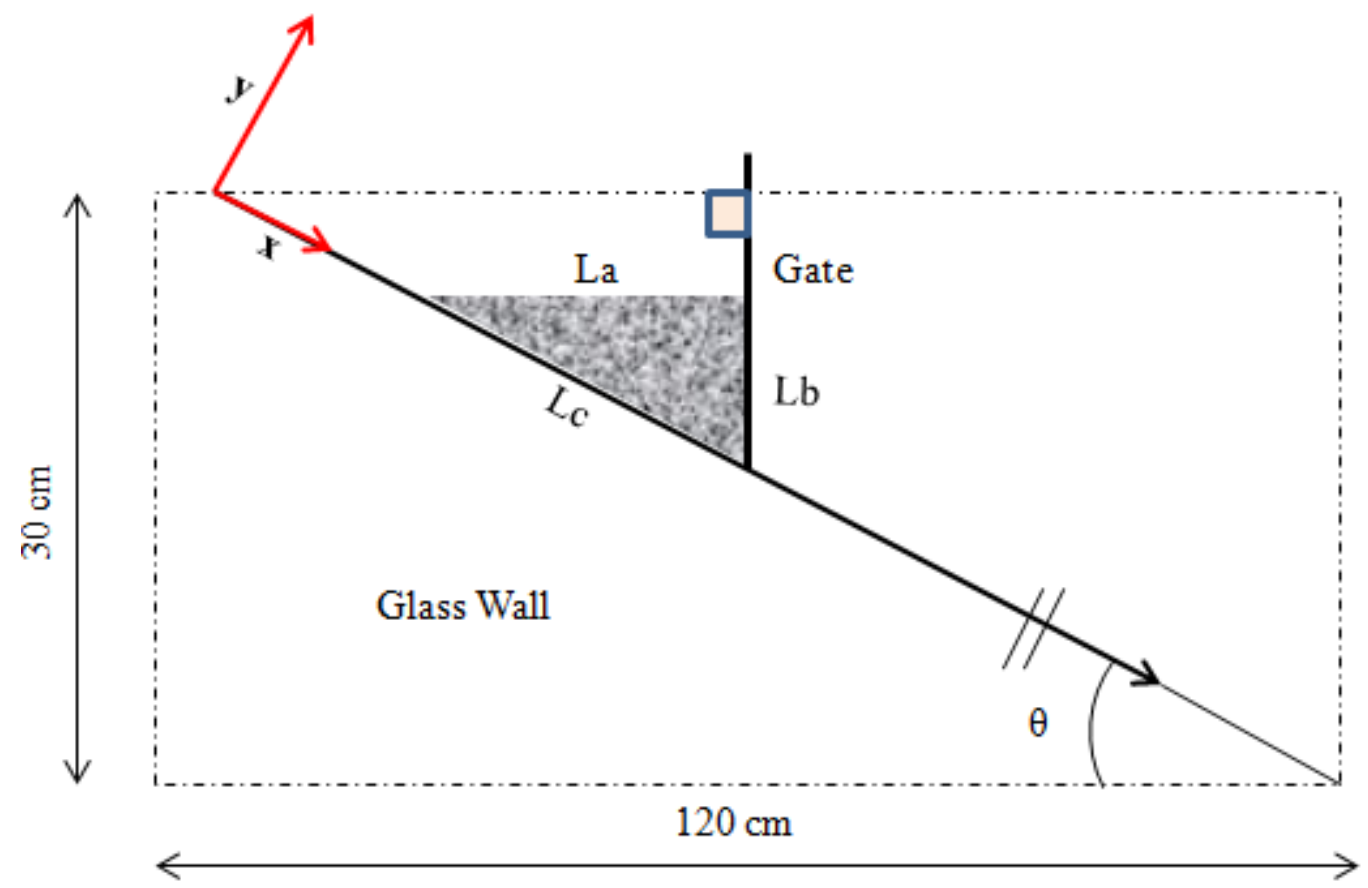

Fig. 2.1: Experimental setup.

\subsection{Evolution of the flow body}

The deformed profile of glass bead granule from the experiment is shown in Figs. 2.2 and 2.3 above for both $45.2^{\circ}$ and $30.8^{\circ}$ slope. This snapshot has been rotated to the horizontal plane after the processing of the images captured by the high speed camera. The avalanche starts to flow from rest due to the low basal friction and flows infinitely as a result of the flow regime. Due to experimental limitations from the capturing of the rapid flow, only an approximate length is considered in this study. For simplicity, the two angles mentioned above would be referred to as $45^{\circ}$ and $30^{\circ}$ in other sections of this thesis. 

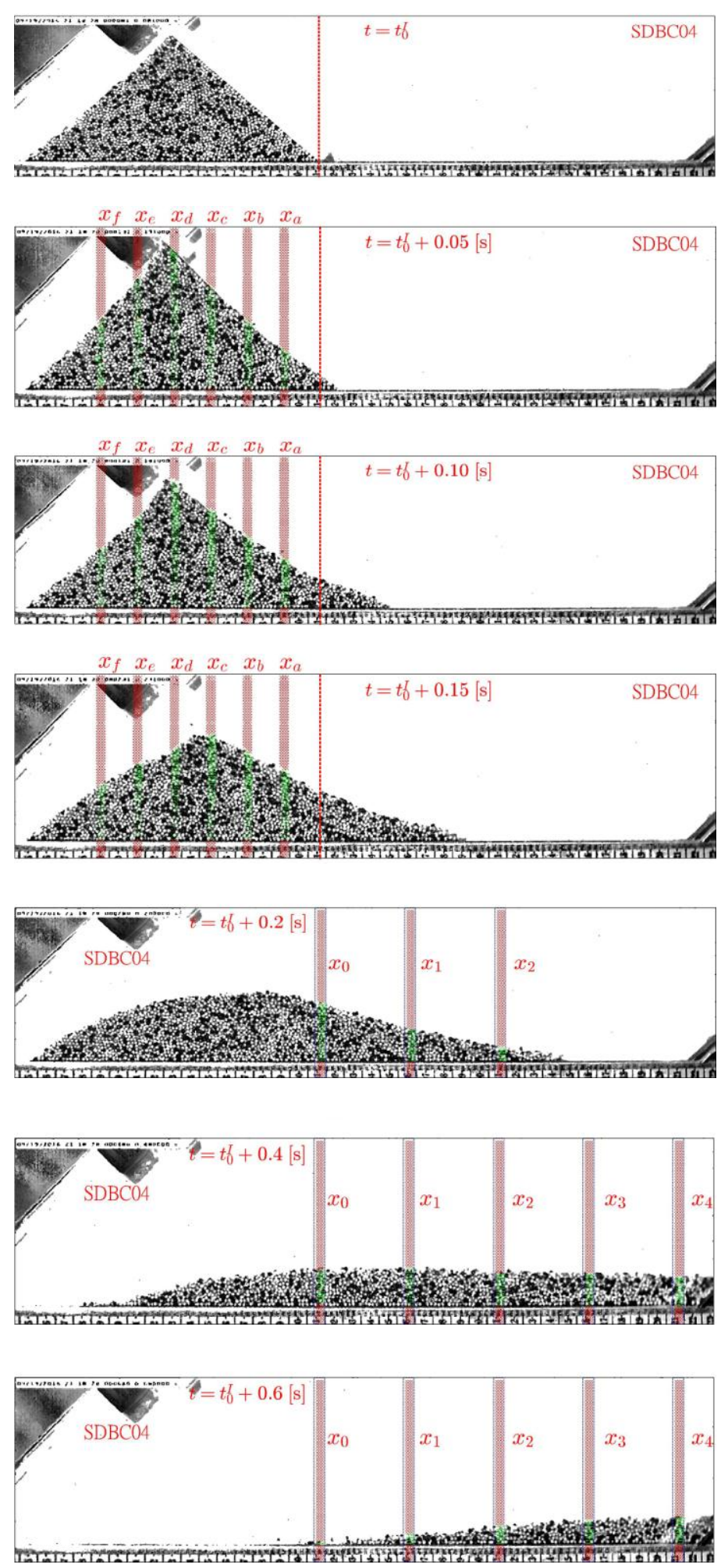

Fig. 2.2: The series of snapshots for an experiment with glass bead granules for $45^{\circ}$ slope.

(The single red dash-line indicates the initial flow front at 0 s while others are selected positions for the velocity profile) 

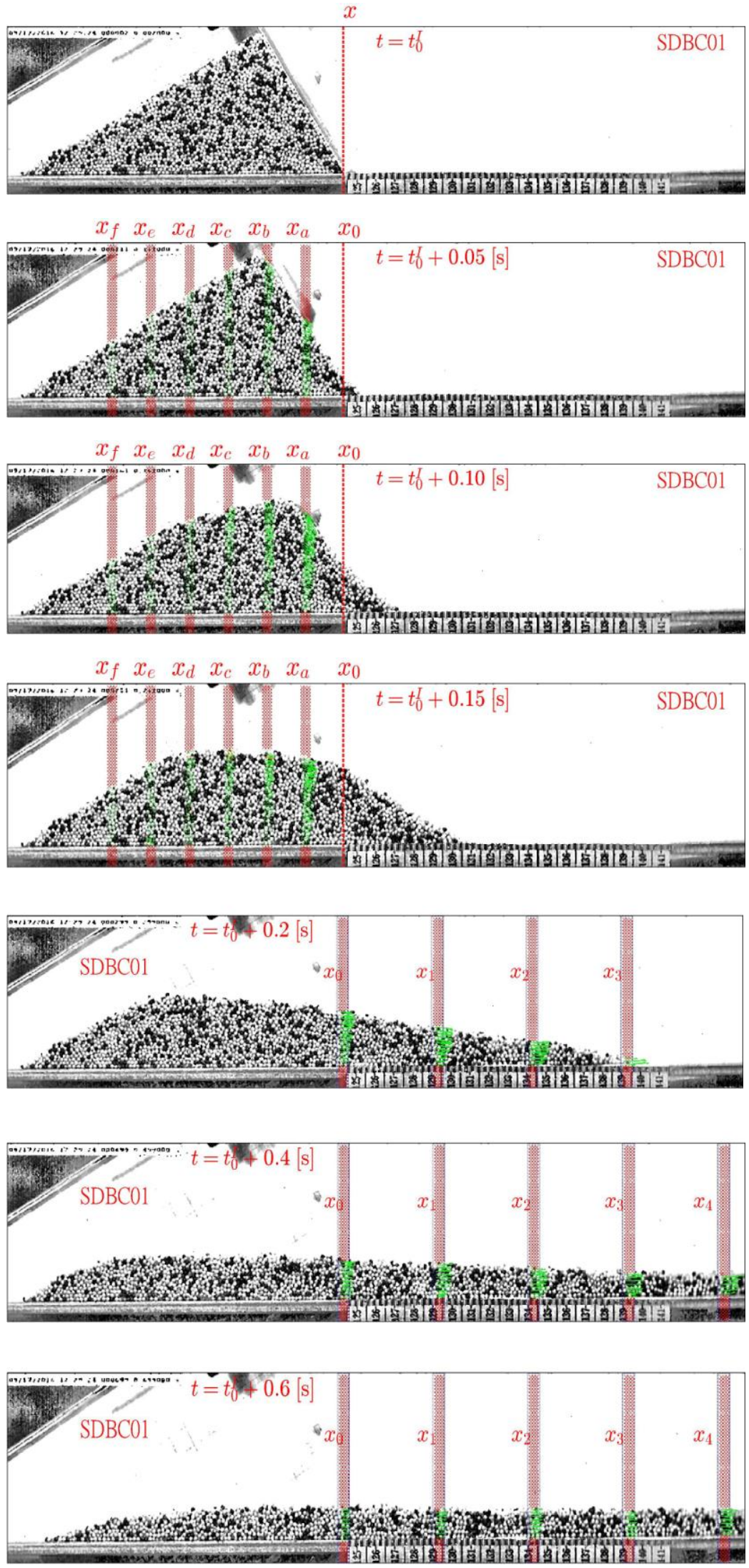

Fig. 2.3: The series of snapshots for an experiment with glass bead granules for $30^{\circ}$ slope. 
It is observed that the initial profile of the granular materials on the inclined plane differs but transits to a similar profile at a later time step. Although the rate of deformation is much slower for the $30^{\circ}$ inclination, it follows similar flow dynamics with the $45^{\circ}$. At the early stages of the granular materials flow $(t=0-0.10 \mathrm{~s})$, the rate of deformation for both angles are observed to be nearly constant before the rate of deformation for the $45^{\circ}$ increased at around $0.2 \mathrm{~s}$. The velocity profiles are averaged at transverse section to properly interpolate missing points for the accuracy of the measured properties. This is shown by the thick red lines while the centers are the measured profile used in this study.

\subsection{Computational conditions}

In this current study, MPS coupled with the local rheology model $\mu(I)$ is employed to validate experimental studies for unsteady granular materials flow. Inclinations greater than the maximum angle of stability were used as shown in Table 2.1 (Silbert et al. 2003) to simulate the deformation of the granular materials on inclined planes.

Table 2.1 Geometry and simulation parameters

Case study parameters

\begin{tabular}{llllll}
\hline Case & $\theta$ & $L a(\mathrm{~cm})$ & $L b(\mathrm{~cm})$ & $L c(\mathrm{~cm})$ & No's of Particles \\
\hline $\mathbf{1}$ & $45.2^{\mathbf{0}}$ & 11.30 & 11.28 & 16.09 & 1711 \\
\hline $\mathbf{2}$ & $30.8^{\mathbf{0}}$ & 14.44 & 08.51 & 16.90 & 1548 \\
\hline \multicolumn{7}{l}{ Simulation parameters } \\
\hline$\theta_{s}$ & $\theta_{2}$ & $\rho_{s}\left(\mathrm{~kg} / \mathrm{m}^{3}\right)$ & $\varepsilon$ & $I_{0}$ & \\
\hline $19.6^{\mathbf{0}}$ & $28.5^{\mathbf{0}}$ & 2500 & 0.6 & 0.279 & \\
\hline
\end{tabular}


The mass of the granular materials was held by a moving gate, and the simulation section considered was similar to the experimental with a span of $0.4 \mathrm{~m}$.

\subsection{Theoretical formulations}

The introduction of several rheology models have led to questions on determining a more efficient model in explaining the behaviour and characteristics of granular flow. Models have been based on theoretical formulations and generated empirical theories from experimental studies. Lagrée et al. (2011) conducted a review on different rheological formulations such as the Bagnold, Bingham, constant friction models. The results showed the effectiveness in the capturing of the dynamics of high transient granular flows using the $\mu(I)$-rheology. In this thesis the $\mu(I)$-rheology model is adopted to study unsteady granular flow on inclined plane.

\subsection{The rheology $\mu(I)$}

The dynamical behaviour of dense granular flow have been described using constitutive law based on the dimensional analysis relating the shear stress to the normal stress with coefficient of friction as the proportionality, a term controlled by the inertia number (Da Cruz et al. 2005; MiDi, 2004; Jop et al. 2006).

$$
I=\frac{\gamma d_{g}}{\sqrt{\frac{P}{\rho_{S}}}} \text { with } \mu(I)=\frac{\tau_{i}}{P}
$$

where $I$, the inertia number is defined as the square root of the Salvage number or Coulomb number (Ancey et al. 1999; Forterre and Pouliquen, 2008; Savage, 1984), 
$\mu(I)$ is the friction coefficient, $P$ is the pressure, $d_{g}$ is the grain size in diameter, $\rho_{s}$ is the density of granular materials, $\tau_{i}$ is the local tangential shear stress, $\gamma_{i j}$ is the strain rate tensor and its second invariant $\gamma$ with $\partial_{j} u_{i}$ and $\partial_{i} u_{j}$ taken to be the partial derivatives of velocity components in the $i$ and $j$ directions as defined below:

$$
\gamma_{i j} \equiv\left(\partial_{j} u_{i}+\partial_{i} u_{j}\right) \quad \gamma=\sqrt{0.5 \gamma_{i j} \gamma_{i j}}
$$

The inertia number is very small for slow flows still in the quasi-static phase and large for rapid flows where the confinement force becomes diluted due to the high shear rates. This dimensional analysis demonstrates the collisional and frictional flow regime. The frictional rheology expressed with the internal stress tensor and phenomenological friction coefficient as expressed by Jop et al. (2006) and Schaeffer, (1987) is given below:

$$
\begin{gathered}
\mu(I)=\mu_{s}+\frac{\mu_{2}-\mu_{s}}{\frac{I_{0}}{I}+1} \quad \text { and } \quad \varepsilon=\varepsilon_{\max }+\left(\varepsilon_{\min }-\varepsilon_{\max }\right) I \\
\sigma_{i j}=-P \delta_{i j}+\tau_{i j,} \text { with } \quad \tau_{i j}=\frac{\mu(I) P}{\gamma} \gamma_{i j}
\end{gathered}
$$

The granular materials only flow if the following condition is satisfied:

$$
|\tau|>\mu_{s} P \text { where } \quad|\tau|=\sqrt{0.5 \tau_{i j} \tau_{i j}}
$$

where the static friction coefficient $\mu_{s}=\tan \theta_{s}$, and the dynamic friction coefficient $\mu_{2}$ $=\tan \theta_{2}$ are material dependent coefficients (note that $\theta_{s}$ and $\theta_{2}$ are static and dynamic 
frictional angles), $I_{0}$ is the empirical coefficient constant, $\tau_{i j}$ is the deviatoric stress, $\sigma_{i j}$ is the Cauchy stress tensor, $\delta_{i j}$ a unit tensor, $\varepsilon$ the volume of fraction. The variation in the volume of fraction of granular particles when shear is applied for large inertia number is also given above. Typical values used are $\varepsilon_{\min }=0.4$ and $\varepsilon_{\max }=0.6$ (Forterre and Pouliquen, 2008; MiDi, 2004) indicating a weak dependence on the inertia number.

\subsection{Regularisation technique}

To minimize the non differential errors from the dimensional analysis relationship, a simple regularisation technique $\eta_{R}$ is used to smoothing the results. Depending on high or low shear approximations, an appropriate small value $\alpha_{r}$ is introduced to yield an order of convergence (Chauchat and Médale, 2014; Frigaard and Nouar, 2005). In this study $\alpha_{r}$ is taken to be 0.000001 as used by Xu and Jin (2016).

$$
\eta_{R}=\left(\mu_{s}+\frac{\left(\mu_{2}-\mu_{s}\right)|\dot{\gamma}|}{I_{0} \sqrt{\varepsilon P}+|\dot{\gamma}|}\right) \frac{P}{\left(|\dot{\gamma}|^{2}+\alpha_{r}^{2}\right)^{\frac{1}{2}}}
$$

The granular flow characteristic is relatively similar to the visco-plastic (Chauchat and Médale, 2014).

\subsection{Governing equations}

The governing equations used for the particle type method are the continuity equation and the Navier-Stokes momentum equation describing the incompressible fluid flow for mass and momentum conservation. This equation is mostly represented in Lagrangian form in the MPS method: 


$$
\begin{gathered}
\frac{D \rho}{D t}=-\rho \nabla \cdot \boldsymbol{u}=0 \\
\frac{\rho D \boldsymbol{u}}{D t}=-\nabla P+\nabla \cdot \tau+\mu \nabla^{2} \mathbf{u}+\rho g
\end{gathered}
$$

Where $\boldsymbol{u}$ is the particle velocity vector, $t$ is time, $g$ denotes the body force which is usually gravity, and $\rho$ represents density for fluid which is converted to the granular materials density $\rho_{s}$ by multiplying the fluid density with the volume fraction, $P$ is pressure and $\tau$ stress tensor. 


\section{CHAPTER 3: NUMERICAL METHOD}

\subsection{MPS method}

Moving Particle Semi-implicit (MPS) method is a gridless approach used to describe the motion of fluid in continuum mechanics based on fully Lagrangian description, where fluid characterization occurs on discrete particle interaction without numerical diffusion. Since its inception in 1996 by Koshizuka and Oka, the method has been devoted to solve several simulation problems across several fields and popularly known for its advancements in computational fluid dynamics. The meshless method for solving partial differential equations involves discrete particle interaction that employs the use of kernels for approximation and interpolation numerically. Particles are scaled to appropriate mapping features with each carrying a physical property. As motion takes place interaction of particles occur and is smoothened with scaled kernel functions whilst updating the properties for surrounding particles. The particle equation is generated based on kernel functions estimated from derivatives of kernel density satisfying certain requirements which are mostly positive definiteness meaning symmetrically positive over the domain which generates metric distances, optimum searching radius, compaction over the domain and unified relative to zero interaction. They are based on summation of physical properties relative to the entire particles in a given domain. Several kernel functions have been used in several studies as shown in Table 3.1. The kernel function $\omega \mathrm{r}_{0}$ adopted in this study is stated below (Shakibaeinia and Jin 2010).

$$
\omega(r)= \begin{cases}\left(1-\frac{r}{r_{e}}\right)^{3}, & 0 \leq \frac{r}{r_{e}}<1 \\ 0, & \frac{r}{r_{e}} \geq 1\end{cases}
$$


Table 3.1 List of other successful weighted functions

\begin{tabular}{|c|c|c|}
\hline $\mathrm{S} / \mathrm{N}$ & Functions & Conditions \\
\hline W1 & $\omega(r)=\left\{\begin{array}{l}\frac{r_{e}}{r}-1 \\
0\end{array}\right.$ & $\begin{array}{l}0 \leq r<r_{e} \\
r_{e} \leq r \\
\text { Koshizuka et al. } \\
(1998)\end{array}$ \\
\hline W2 & $\omega(r)=\left\{\begin{array}{l}\frac{40}{7 \pi r_{e}^{2}}\left(1-6\left(\frac{r}{r_{e}}\right)^{2}+6\left(\frac{r}{r_{e}}\right)^{3}\right) \\
\frac{10}{7 \pi r_{e}^{2}}\left(2-2\left(\frac{r}{r_{e}}\right)\right)^{3} \\
0\end{array}\right.$ & $\begin{array}{l}0 \leq r<\frac{r_{e}}{2} \\
\frac{r_{e}}{2}<r<r_{e} \\
r>r_{e} \\
\text { Shao and Lo } \\
\text { (2003) }\end{array}$ \\
\hline W3 & $\begin{array}{l}\omega(r) \\
=\left\{\begin{array}{l}\frac{480 \sqrt{2}-705}{512 \sqrt{2}-745} \frac{1}{r_{e}}+\frac{-960 \sqrt{2}-1515}{512 \sqrt{2}-745} \frac{1}{r_{e}}\left(\frac{r}{r_{e}}\right)^{2}+ \\
\frac{-210}{512 \sqrt{2}-745} \frac{1}{r_{e}}\left(\frac{r}{r_{e}}\right)^{2}+\frac{480 \sqrt{2}-600}{512 \sqrt{2}-745} \frac{1}{r_{e}}\left(\frac{r}{r_{e}}\right)^{4}\end{array}\right.\end{array}$ & $\begin{array}{l}0 \leq r \leq r_{e} \\
r_{e}<r \\
\text { Sheu et al. } 2011\end{array}$ \\
\hline W4 & $\omega(r)=\left\{\begin{array}{l}\left(1-\frac{r}{r_{e}}\right)^{3} \quad\left(1+\frac{r}{r_{e}}\right)^{3} \\
0\end{array}\right.$ & $\begin{array}{l}0 \leq r<r_{e} \\
r_{e}<r \\
\text { Kyung et al. } \\
2014\end{array}$ \\
\hline W5 & $\omega(r)=\left\{\begin{array}{l}\frac{r_{e}}{0.85 r+0.15 r_{e}}-1 \\
0\end{array}\right.$ & $\begin{array}{l}0 \leq r<r_{e} \\
r_{e} \leq r \\
\text { Zhang and Wan } \\
2012\end{array}$ \\
\hline
\end{tabular}




\subsubsection{Requirement of the Kernel Function}

The two major requirements for smoothing functions used in the mps method are stated below based on literature reviews (Tony et al. 2011 and Souto-Iglesias et al. 2013) and are used to determine the appropriate kernel function to be used in this present study.

Property 1

The smoothing function should satisfy the smoothed Dirac delta function also sometimes called nascent delta functions. This function can also be viewed as a limit Gaussian or a Lorentzian.

$$
\lim _{r_{e} \rightarrow 0} \omega\left(r, r_{e}\right) d r=1 \quad \ldots \quad \int_{-\infty}^{\infty} \omega\left(r, r_{e}\right) d r=1
$$

\section{Property 2}

The smoothing function should satisfy the Mercer's condition stated as positivedefinite symmetric.

The ratio between the relative distance between particles and the searching radius is to scale the physical parameters of the kernel functions respective to the particle interaction range. Fig. 3.1 below shows the relationship between the kernels and the scaled ratio of the kernels listed on Table 3.1.

In this current study the weighted function employed is a third-order polynomial spiky kernel function developed by Shakibaeinia and Jin (2010), which is defined as taken as $\omega \mathrm{r}_{0}$ from Equation 3.1. Although $\omega \mathrm{r}_{0}$ and $\mathrm{W} 4$ meet the stated conditions for the smoothing function, $\omega \mathrm{r}_{0}$ is said to prevent clustering of particles and avoids an infinity value when neighboring particles are relatively close to each other (Shakibaeinia and Jin, 2010). 


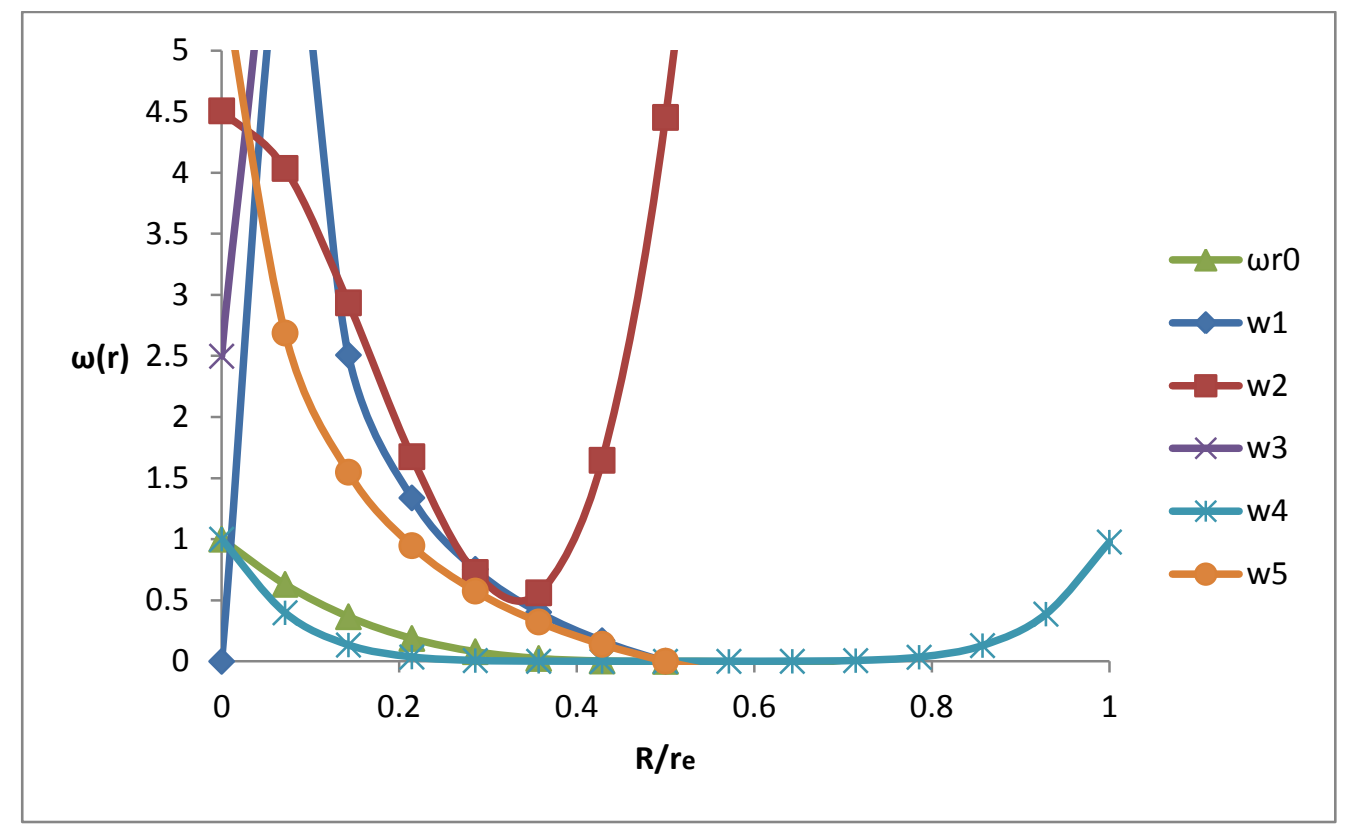

Figure 3.1: Plotted kernel functions from table 3.1 
where $\omega(r)$ the kernel function, $r_{e}$ is the radius of the interaction circle around each targeted particle, taken as $4.0 d_{l}$ ( $d_{l}$ is the particle size) and $r$ is the distance between a target particle $i$ and one of its neighboring particles $j$ as shown below:

$$
r=\sqrt{\left|x_{j}-x_{i}\right|^{2}+\left|y_{j}-y_{i}\right|^{2}}
$$

The particle number density at a position $i$ is defined as follows

$$
\langle n\rangle_{i}=\sum_{j \neq i} \omega\left(\left|r_{j}-r_{i}\right|, r_{e}\right)
$$

Assuming a constant mass for all particles, the relationship between the particle number density and real fluid density is stated below:

$$
\langle\rho\rangle_{i}=\frac{\sum_{j \neq i} m_{i} \omega\left(\left|r_{j}-r_{i}\right|, r_{e}\right)}{\int_{v} \omega\left(\left|r_{j}-r_{i}\right|, r_{e}\right) d v}
$$

where $m_{i}$ is the mass of the fluid particle, hence the continuity equation is satisfied when the particle number density is kept constant.

\subsection{Discretization technique}

The interaction of particles with the help of the kernel function is used define the gradient and Laplacian operator with the use of arbitrary scalar term $\phi$ (Koshizuka and Oka, 1996) as expressed below.

$$
\begin{gathered}
\left.\langle\nabla \phi\rangle\right|_{i j}=\frac{d}{n^{0}} \sum_{j \neq i} \frac{\left(\phi_{j}-\phi_{i}\right)}{\left|\vec{r}_{j}-\vec{r}_{l}\right|^{2}}\left(\vec{r}_{j}-\vec{r}_{l}\right) \omega\left(\vec{r}_{j}-\vec{r}_{l}\right) \\
\left.\left\langle\nabla^{2} \phi\right\rangle\right|_{i j}=\frac{2 d}{\lambda n^{0}} \sum_{j \neq i}\left(\phi_{j}-\phi_{i}\right) \omega\left(\vec{r}_{j}-\vec{r}_{l}\right)
\end{gathered}
$$


where $n^{0}$ can also be taken as $n_{i}$ under the incompressible flow condition and $\lambda$ is a coefficient used to scale the variance for consistency with the analytical given below (Koshizuka and Oka, 1996):

$$
\lambda=\frac{\int_{v} \omega\left(\left|\vec{r}_{j}-\vec{r}_{l}\right|\right)\left|\vec{r}_{j}-\vec{r}_{i}\right|^{2} d v}{\int_{v} \omega\left(\left|\vec{r}_{j}-\vec{r}_{l}\right|\right) d v} \cong \frac{\sum_{j \neq i} \omega\left(\left|r_{j}-r_{i}\right|\right)\left|\vec{r}_{j}-\vec{r}_{l}\right|^{2}}{\sum_{j \neq i} \omega\left(\left|r_{j}-r_{i}\right|\right)}
$$

To calculate the pressure field in the MPS model, the equation of state which was first introduced by Monaghan (1994) to reduce the computing time from computing the pressure term by replacing the fluid density with particle number density as expressed below.

$$
p_{i}=\frac{\rho c_{0}^{2}}{\zeta}\left(\left(\frac{\langle n\rangle_{i}^{*}}{n^{0}}\right)^{\varsigma}-1.0\right)
$$

where $\zeta$ is a coefficient taken as 7 (Monaghan 1994, Shakibaeinia and Jin 2010), $c_{0}$ is the artificial sound speed in the reference medium. The limitation of the compressibility of the granular materials to less than $1 \%$ is based on the artificial sound of speed at 10 times the maximum flow velocity.

\subsection{Boundary conditions}

At the free surface no particle exist outside the region, the particle number density decreases for particles on the free surface. To identify the free surface particles, the condition stated below must be satisfied.

$$
\langle n\rangle_{i}^{*}<\beta n^{0}
$$

where $\beta$ is the threshold coefficient, it value ranges between 0.8 and 0.99 (Shakibaeinia and Jin 2010). In this study $\beta$ is taken as 0.99. A similar approach is 
used for the solid boundary where several evenly distributed ghost particles are added beyond every solid particle to avoid density deficiency close to the boundary. The radius of interaction of particles with a minimum of four layers of ghost particles are used beyond all solid boundary sections in this study to enable more interaction between particles within the searching radius a study also conducted by (AtaieAshtiani, B., and Farhadi, 2006). Throughout the simulation, the fluid, solid and ghost particles are the three particle types used while maintaining a constant particle searching radius.

Also in calculating the motion of particles and velocity, a predictor and corrector approach is employed to solve the governing equation. A prediction is made regarding particle positions and velocity $\mathrm{u}^{*}$ and $\mathrm{r}^{*}$ resulting to $\mathrm{n}^{*}$ which is a particle number density using the new location from the temporal velocity and position of particles as shown below:

$$
\begin{gathered}
u^{*}=u^{n}+\Delta t(\nabla \tau+f) \\
r^{*}=r^{n}+u^{*} \Delta t \\
\langle n\rangle_{i}^{*}=\sum_{j \neq i} \omega\left(\left|r_{j}-r_{i}\right|^{*}, r_{e}\right)
\end{gathered}
$$

The velocity and particle correction is then stated below for new particles

$$
\begin{gathered}
u^{n+1}=u^{*}-\frac{\Delta t}{\rho} \nabla p^{n+1} \\
r^{n+1}=r^{n}+u^{n+1} \Delta t .
\end{gathered}
$$

where $\Delta t$ is taken as the time step, depending on the numbers of particles the above is calculated for all particles at every time steps. 


\section{CHAPTER 4: RESULT AND DISCUSSION}

\subsection{Properties of the granular flow behaviour}

Experimental studies from the instantaneous collapse of the glass beads on inclined planes were used to validate results from numerical model, while extensive microscopic observations beyond the scope of physical measurements were captured and further discussed using the numerical model.

\subsection{Velocity profile}

The rheology model is applied to simulate unsteady granular flows on inclined planes. Inclinations greater than the angle of repose $\theta_{r}$ are used to quantitatively predict the velocity profile by simulation and physical observations. Although both experimental setups and numerical configuration are made identical, the effect of the sliding gate leading to instantaneous effect of gravity differ as granular particles close to the gate deflects upward with larger velocities in the simulation compared to the experimental observation. This led to fluctuations at the wave front locations at time $0.05 \mathrm{~s}$ at both inclination angles for the numerical results due to the treatment of the interface between the sliding gate and the granular material. Figs. $4.1-4.4$ shows the horizontal and vertical velocity profile for $45^{\circ}$ and $30^{\circ}$ slope where the flow condition is $\theta_{s}<\theta, \theta>\theta_{2}$. The dense nature of the granular flow is maintained for a very short time before transiting into a dilute state with the front region becoming agitated. The dense to dilute state can be distinguished based on the vector interaction between neighbouring particles in the MPS method. Dimensionless parameters $\stackrel{x}{u}=u / \sqrt{ } g d_{g}, \stackrel{x}{v}$ $=v / \sqrt{ } g d_{g}$ and $\stackrel{x}{y}=y / d_{g}$ are used to denote both the horizontal and vertical components 
of the velocity and the position of the granular materials during deformation, where $g$ is acceleration due to gravity and $d_{g}$ is the grain size. To describe the granular flow dynamics and profile, the positions where the depth of the granular flow $(h)$ is observed to be at its peak are selected from the rear, front, and the intermediate section of the shearing granular materials. Granular particles located before $0.108 \mathrm{~m}$ remained in a quasi-static state with little variation in the surface profile caused by the differentials in the rate of deformation of the granular materials at $0.05 \mathrm{~s}$ as shown in Fig. 4.1. At $0.108 \mathrm{~m}$ the increase in the plasticity effect leads to a convex shape profile similar to observations from (Ancey, 2001; Jop et al. 2006; MiDi, 2004; Schaefer, 1987; Schaefer et al. 2010). Also there exists a slight increase in the free surface depth at $0.05 \mathrm{~s}$ at location $0.148 \mathrm{~m}$, this is predicted to have occurred from the different boundary conditions found in the experiment and the assumed for the simulation as also stated in (Pouliquen, 1999; Silbert et al. 2003). The velocity profile transition is observed from the quasi-static state into a developed granular flow with trends of a power law and gradually into a linear relationship between $\stackrel{x}{u}$ and $\stackrel{x}{y}$. The temporal and deformation of the vertical velocity profile at $45^{\circ}$ inclination plane is illustrated in Fig. 4.2. At $t=0.05 \mathrm{~s}$, a quasi static state can be observed in Fig. 4.2a, with gradual deviation of the granular materials from location $x=0.108 \mathrm{~m}$ approximately midway of the depth profile. 
(a) $\quad t=0.05 \mathrm{~s} x=0.048 \mathrm{~m}$

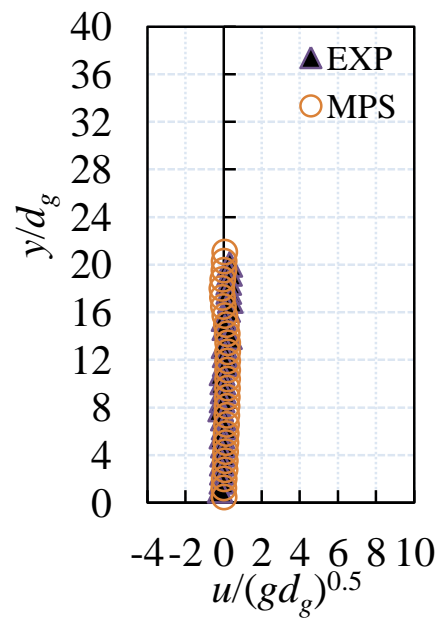

(d) $t=0.2 \mathrm{~s} x=0.168 \mathrm{~m}$

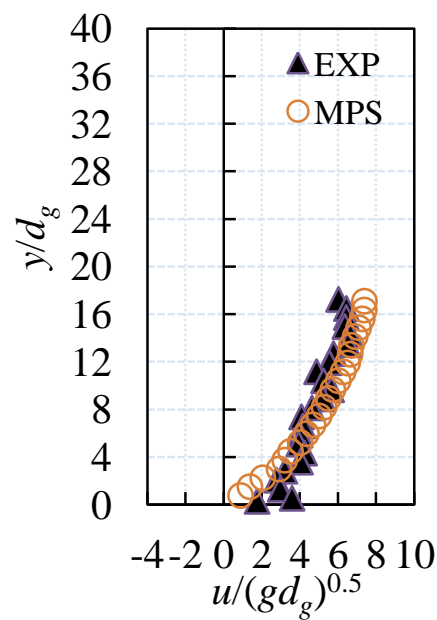

(g) $t=0.4 \mathrm{~s} x=0.168 \mathrm{~m}$

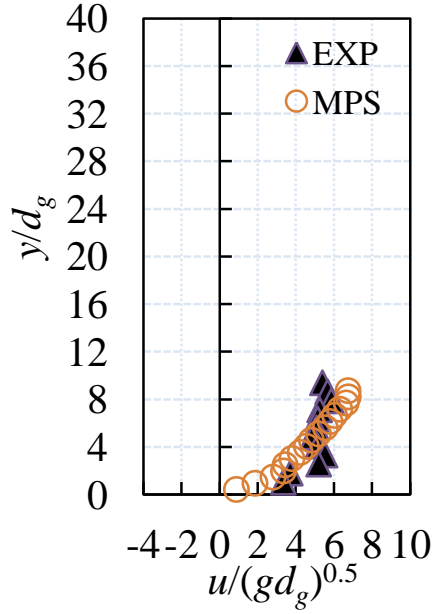

(b) $t=0.05 \mathrm{~s} x=0.108 \mathrm{~m}$

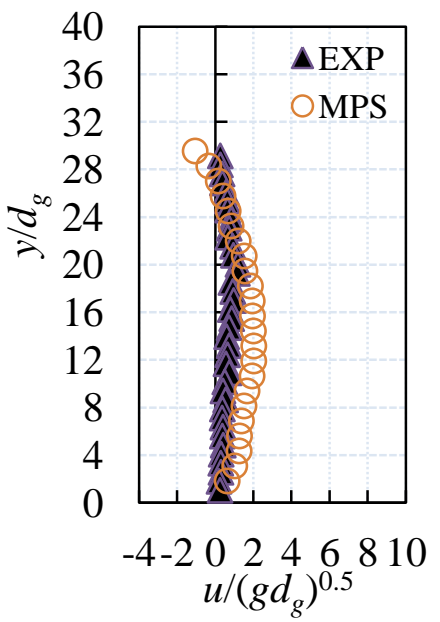

(e) $t=0.2 \mathrm{~s} x=0.218 \mathrm{~m}$

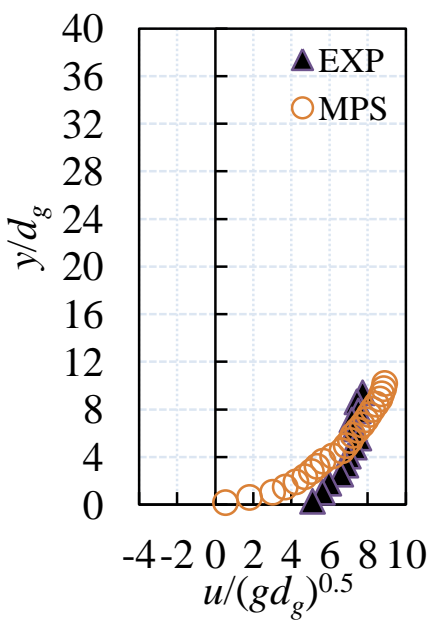

(h) $t=0.4 \mathrm{~s} x=0.218 \mathrm{~m}$

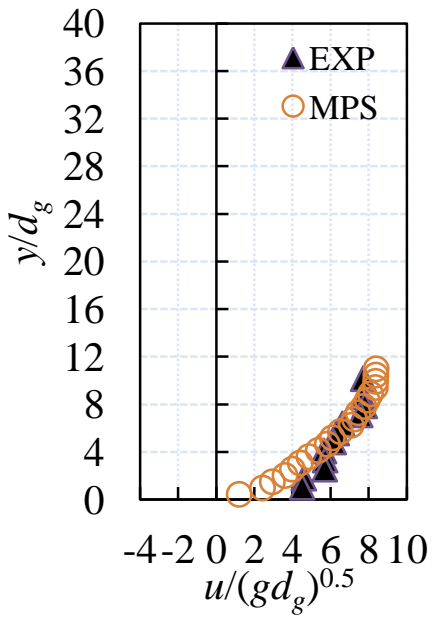

(c) $t=0.05 \mathrm{~s} x=0.148 \mathrm{~m}$

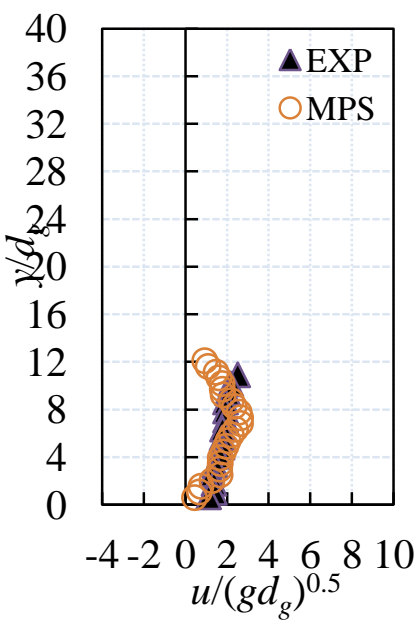

(f) $t=0.2 \mathrm{~s} x=0.268 \mathrm{~m}$

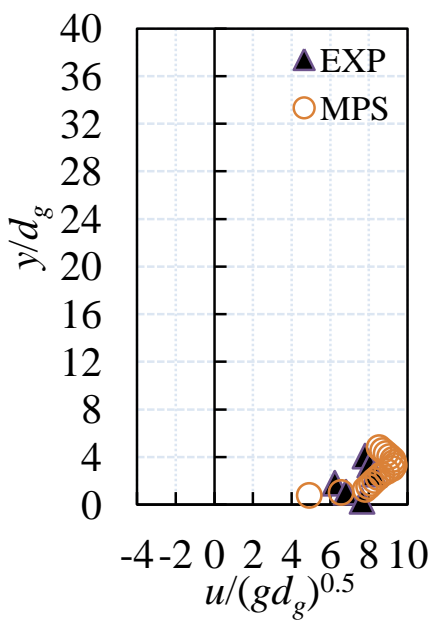

(i) $t=0.4 \mathrm{~s} x=0.268 \mathrm{~m}$

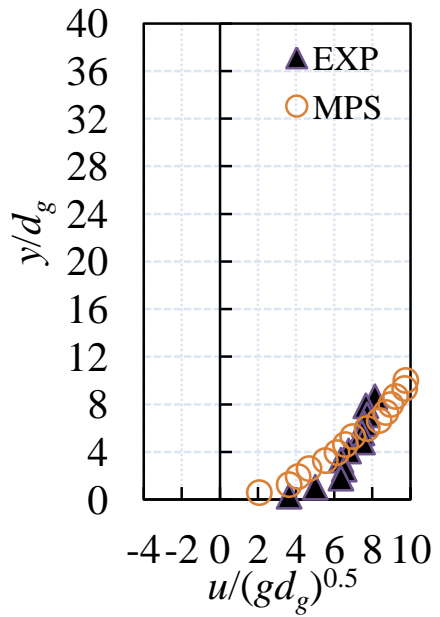

Fig. 4.1 Horizontal velocity profile for $45^{\circ}$ slope showing comparison between experimental and numerical results at a time $t(\mathrm{~s})$ from specific locations 
(a) $t=0.05 \mathrm{~s} x=0.048 \mathrm{~m}$

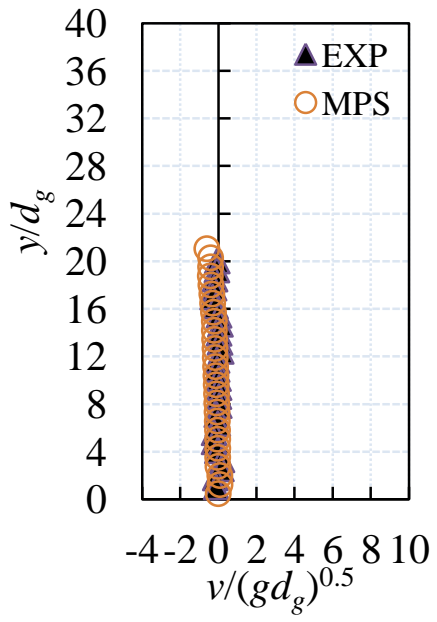

(d) $t=0.2 \mathrm{~s} x=0.168 \mathrm{~m}$

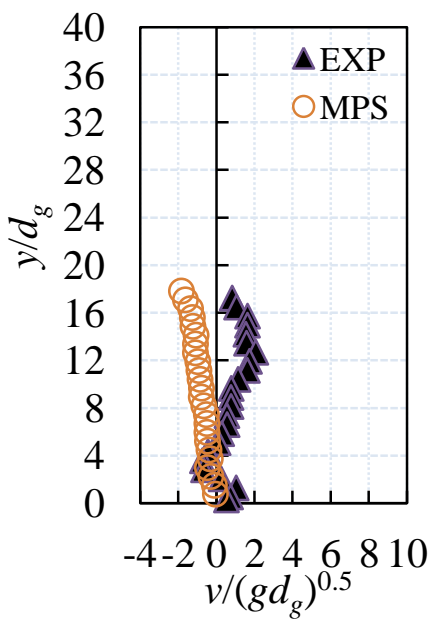

(g) $t=0.4 \mathrm{~s} x=0.168 \mathrm{~m}$

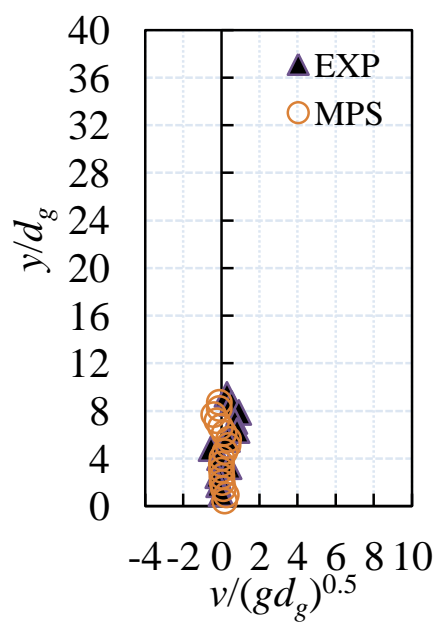

(b) $t=0.05 \mathrm{~s} x=0.108 \mathrm{~m}$

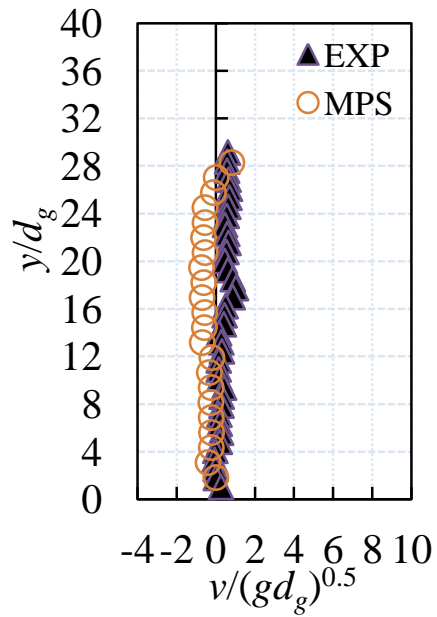

(e) $t=0.2 \mathrm{~s} x=0.218 \mathrm{~m}$

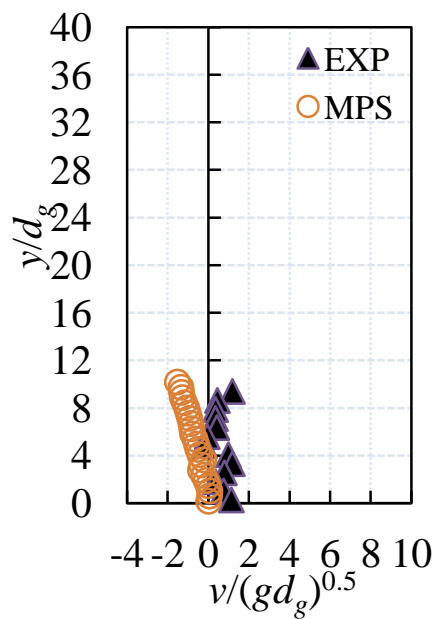

(h) $t=0.4 \mathrm{~s} x=0.218 \mathrm{~m}$

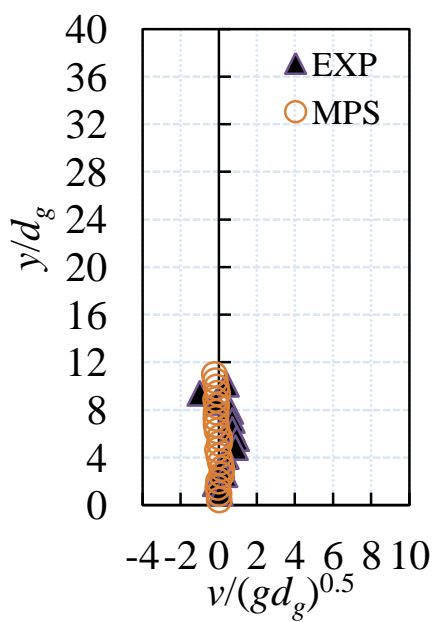

(c) $t=0.05 \mathrm{~s} x=0.148 \mathrm{~m}$

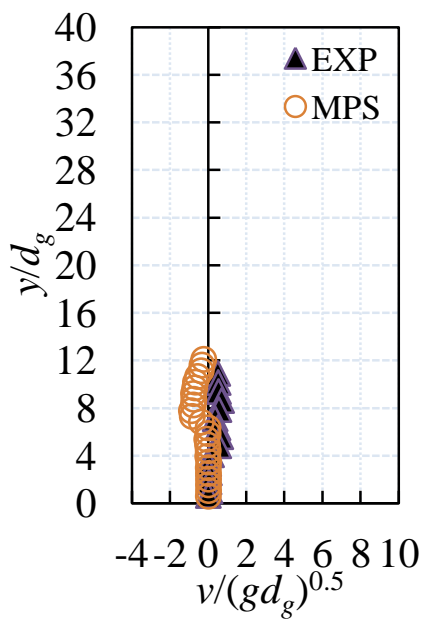

(f) $t=0.2 \mathrm{~s} x=0.268 \mathrm{~m}$

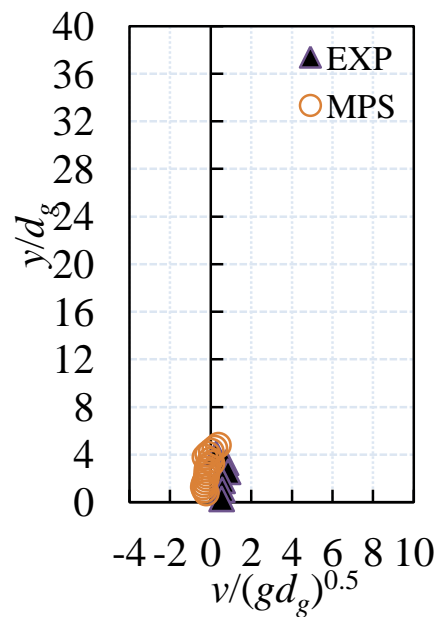

(i) $t=0.4 \mathrm{~s} x=0.268 \mathrm{~m}$

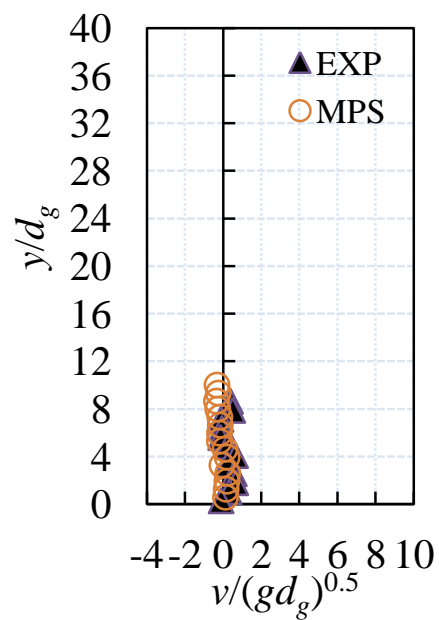

Fig. 4.2 Vertical velocity profile for $45^{\circ}$ slope showing comparison between experimental and numerical results at a time $t(\mathrm{~s})$ from specific locations 
(a) $t=0.05 \mathrm{~s} x=0.055 \mathrm{~m}$

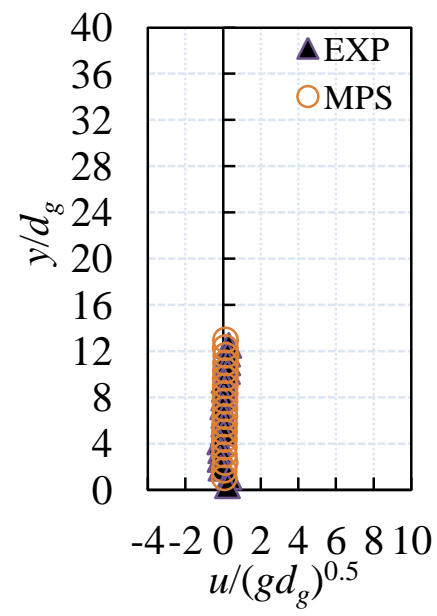

(d) $t=0.2 \mathrm{~s} x=0.179 \mathrm{~m}$

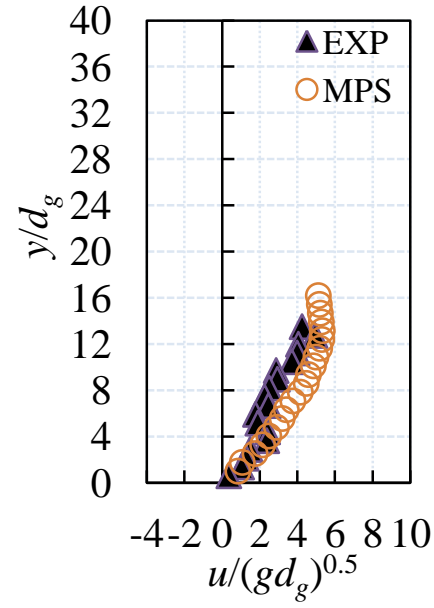

(g) $t=0.4 \mathrm{~s} x=0.179 \mathrm{~m}$

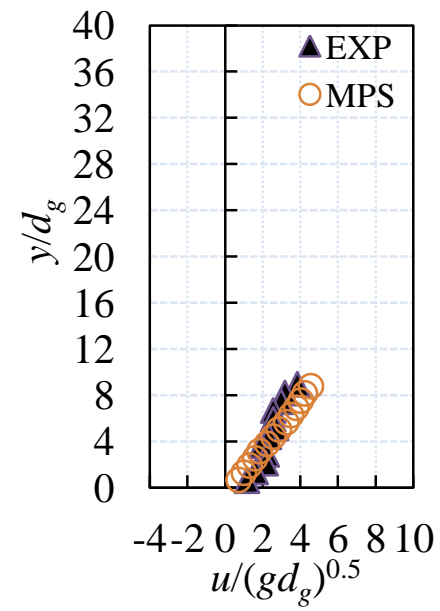

(b) $t=0.05 \mathrm{~s} x=0.115 \mathrm{~m}$

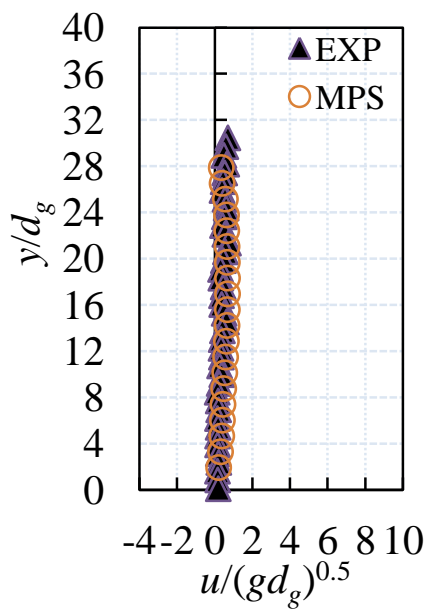

(e) $t=0.2 \mathrm{~s} x=0.229 \mathrm{~m}$

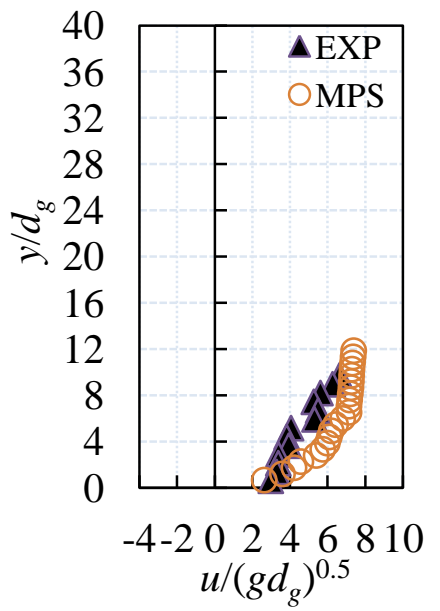

(h) $t=0.4 \mathrm{~s} x=0.229 \mathrm{~m}$

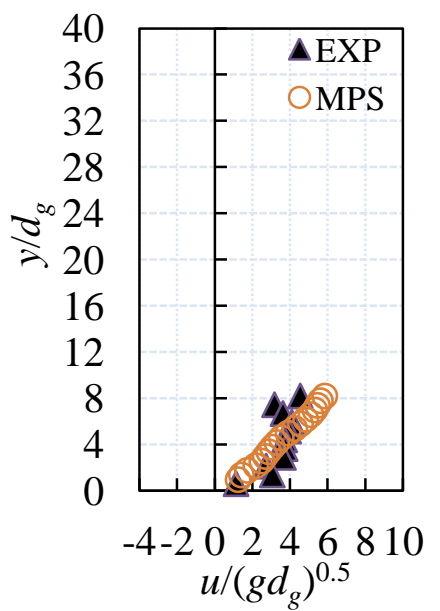

(c) $t=0.05 \mathrm{~s} x=0.15 \mathrm{~m}$

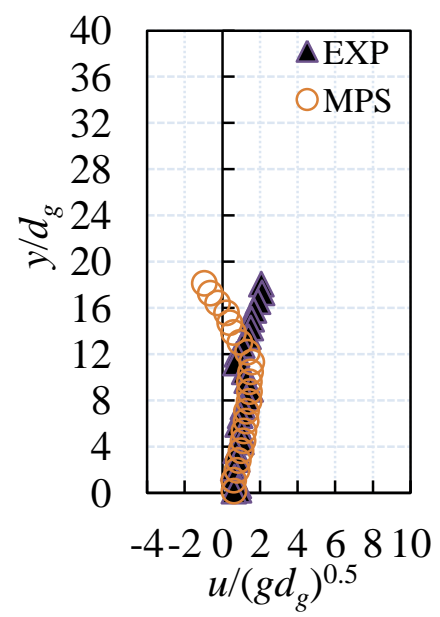

(f) $t=0.2 \mathrm{~s} x=0.279 \mathrm{~m}$

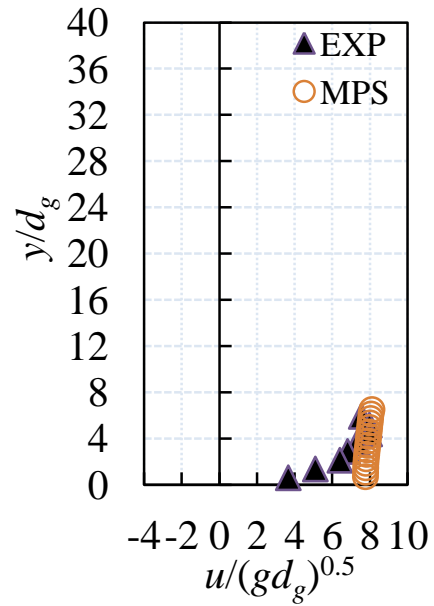

(i) $t=0.4 \mathrm{~s} x=0.279 \mathrm{~m}$

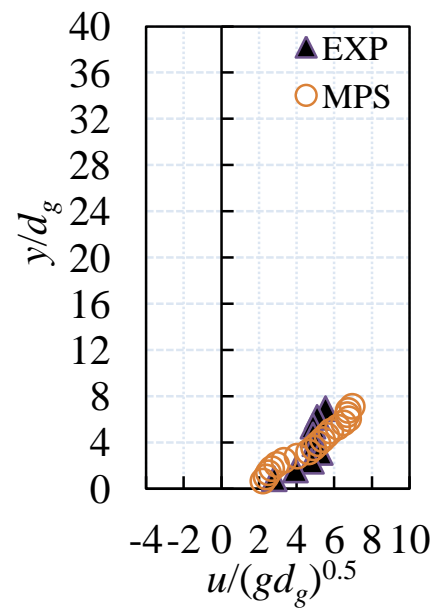

Fig. 4.3 Horizontal velocity profile for $30^{\circ}$ slope showing comparison between experimental and numerical results at a time $t(\mathrm{~s})$ from specific locations 
(a) $t=0.05 \mathrm{~s} x=0.055 \mathrm{~m}$

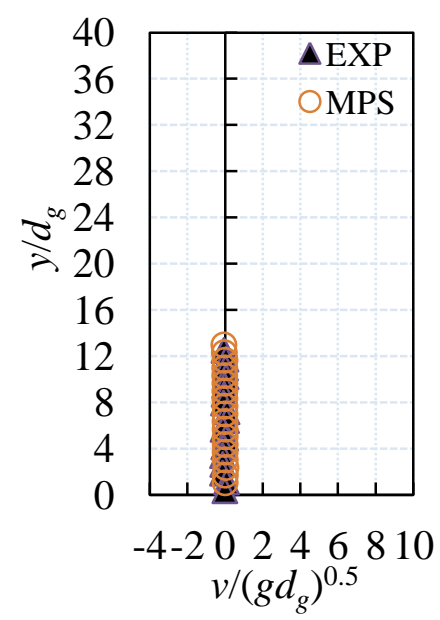

(c) $t=0.2 \mathrm{~s} x=0.179 \mathrm{~m}$

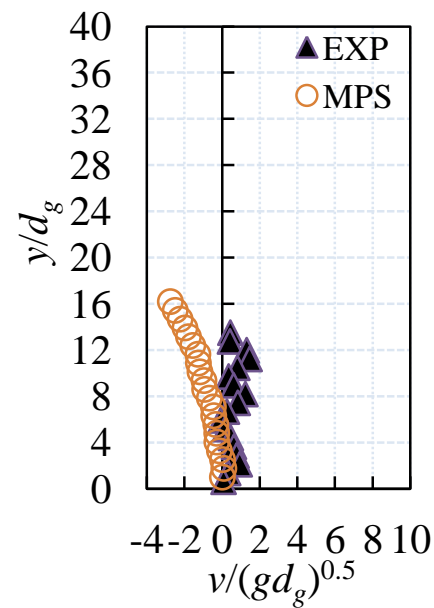

(f) $t=0.4 \mathrm{~s} x=0.179 \mathrm{~m}$

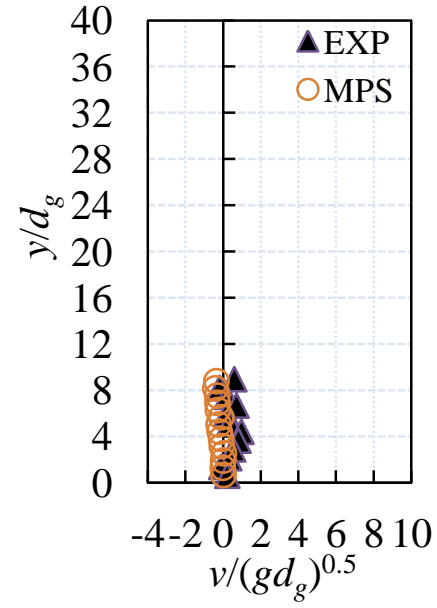

(b) $t=0.05 \mathrm{~s} x=0.115 \mathrm{~m}$

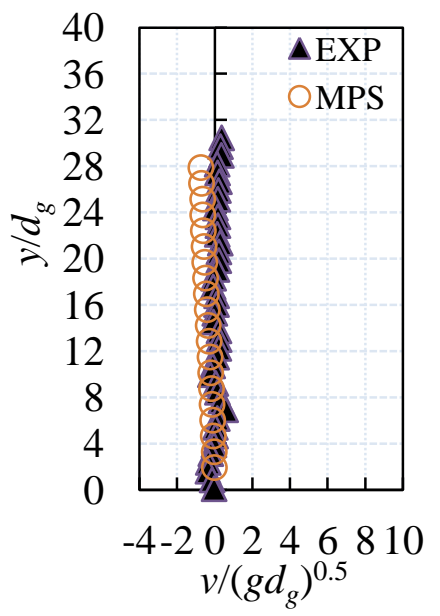

(d) $t=0.2 \mathrm{~s} x=0.229 \mathrm{~m}$

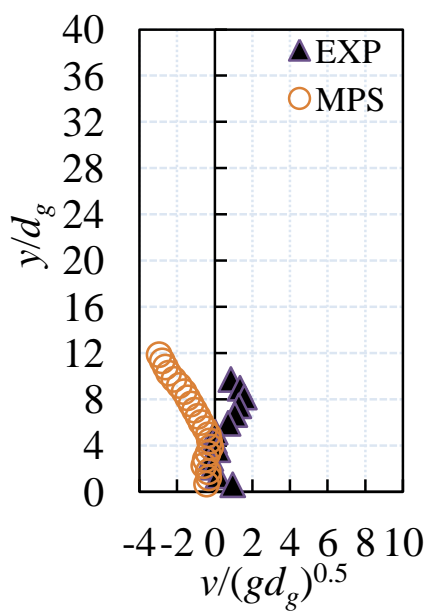

(g) $t=0.4 \mathrm{~s} x=0.229 \mathrm{~m}$

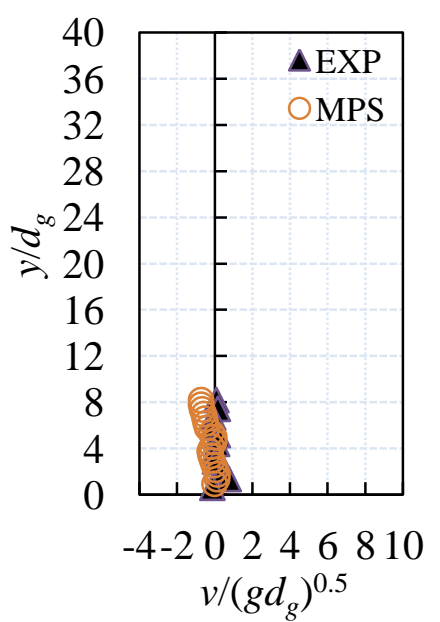

(c) $t=0.05 \mathrm{~s} x=0.15 \mathrm{~m}$

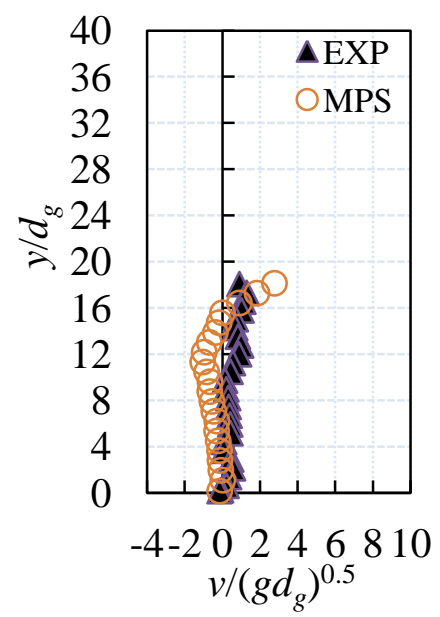

(e) $t=0.2 \mathrm{~s} x=0.279 \mathrm{~m}$

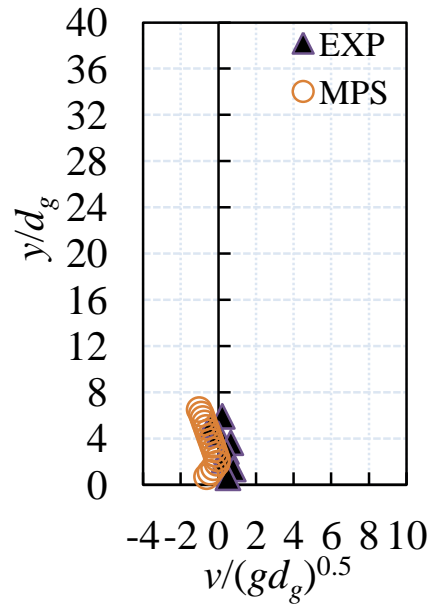

(h) $t=0.4 \mathrm{~s} x=0.279 \mathrm{~m}$

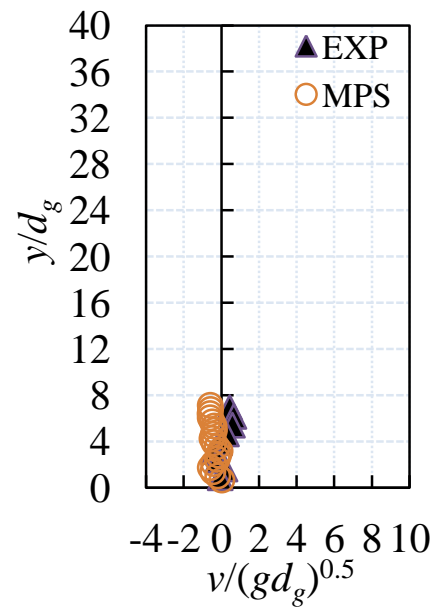

Fig. 4.4 Vertical velocity profile for $30^{\circ}$ slope showing comparison between experimental and numerical results at a time $t(\mathrm{~s})$ from specific locations 
The velocities of the physical study remained positive maintaining only the directional change close to the boundary with that of the MPS remaining in the negative path, meaning no change of direction asides the one which occurred close to the boundary. This change in direction is caused by the effect of gravitational force and the $v_{y}$ being zero at the surface profile and at region where the granular materials is highly dilated. The granular flow is observed to be continuous with the minimum flow depth $h_{\min }$ after full transition to the maximum velocity region becomes uniform until the end of the channel is reached. Particles at location $0.048 \mathrm{~m}$ are maintained in the quasi-static phase. At $0.2 \mathrm{~s}$ at location $0.168 \mathrm{~m}$, the velocity profiles take on a concave shape close to the boundary and after the inflection point to a convex-like shape for the physical observation and a linear trend for the MPS. At location $0.218 \mathrm{~m}$, MPS and the physical observation both display a concave shape with varying velocity distribution. At $0.4 \mathrm{~s}$, the velocity distribution reduces uniformly close to zero before other neighbouring materials collision effects replaces the granular particles at the frontal region of the granular flow. Fig. 4.3 shows that after the opening of the sliding gate at $0.05 \mathrm{~s}$ particles located after $0.095 \mathrm{~m}$ started accelerating slowly while the one before it remained in quasi-static state for $30^{\circ}$ slope. Granular particles located above the boundary region close to the surface tend to attain more magnitude throughout the granular flow. At location $0.15 \mathrm{~m}$ the MPS direction changed around a depth close to the surface profile, while the physical observation maintained the same direction from the boundary with little variation in the uniform velocity distribution. The depth of the granular materials for both physical and MPS at $0.05 \mathrm{~s}$ are well predicted unlike that of $0.2 \mathrm{~s}$ when the flow is developed with the magnitude of the velocity $v_{x}$ increases along the channel at different fixed positions. Decrease in the inclination angle results into reduction in 
the shear rate of the granular materials as observed by comparing $30^{\circ}$ to $45^{\circ}$. This decrease results into longer time for the quasi-static region to transit to the kinetic phase. At $0.05 \mathrm{~s}$, only the wave front region shows visible deformation while other region before $0.115 \mathrm{~m}$ remained in a static. After $0.2 \mathrm{~s}$ for positions greater than $0.279 \mathrm{~m}$ the MPS transits to an approximate uniform velocity distribution with a linear relationship while the physical study maintained a power law trend resulting from discrepancies in the velocity distribution close to the boundary. After the flow is developed at $0.2 \mathrm{~s}$, the physical observation at the rear of the flow have a convex shape and after the inflection point shows a linear relationship while the numerical show a convex shape at locations $0.179 \mathrm{~m}$ and $0.229 \mathrm{~m}$ before showing a linear trend at the wave front region at location $0.279 \mathrm{~m}$. At time $0.4 \mathrm{~s}$, the velocity distribution behaves linearly and also tends to a power law. The physical study shows a concave shape after the inflection point close to the surface profile. This is predicted to have occurred from the instabilities found in the flow. Fig. 4.4 shows good prediction during quasi-static state aside at the peak region of the flow at $0.05 \mathrm{~s}$ from $0.15 \mathrm{~m}$ where the $v_{y}$ component for MPS changed direction towards the boundary and finally returned positive at the surface profile resulting to a concave shape from a depth of $0.025 \mathrm{~m}$ during inflection with a linear trend from the boundary. The depth of the granular materials at this state is approximately equal until time $0.2 \mathrm{~s}$ when the collision interaction between particles differs in resultant direction resulting from the difference in the densely packed neighbouring particles interaction. At 4.0s, the $v_{y}$ for both cases shows good prediction with similar projection. The $45^{\circ}$ and $30^{\circ}$ slopes are observed to share similar properties with very little variations giving good prediction with physical observation. In general it can be observed that the horizontal velocity gave better prediction when compared to the vertical velocity. This may result from 
the influence of gravity from the vertical direction and the assumption of the $\mathrm{z}$ component of the coordinates to be zero.

\subsection{Velocity evolution}

The study of the flow phenomena at transverse fixed position along the channel as the velocity distribution changes with time is illustrated in Figs. 4.5 and 4.6 for the experimental and numerical studies. The selected locations are fixed positions along the channel at the rear, front and intermediate regions of the flowing granular materials. The time selected shows the flow phenomena during the time of the initial development and transition to a fully developed flow phase with limited instabilities. Fig. 4.5a shows that at $t=0.05 \mathrm{~s}-0.15 \mathrm{~s}$, the velocity increased linearly from the boundary to about $70 \%$ reduction in the flow depth. At $t=0.15 \mathrm{~s}$, after the flow is initiated and where slippage of the granular materials is partially developed, the velocity magnitude increases at every corresponding locations. Increase in the velocity magnitude at the peak depth of the granular materials flow is predicted to continue until the flow depth or thickness reduces to minimum $h_{\min }$ at fixed location. At $x=0.148 \mathrm{~m}$ close to the flow front of the flowing granular materials, the magnitude of the velocity reduced indicating that the driving force of the flow is minimal at the location close to the end of the quasi-static phase. For the two locations ( $x=0.048 \mathrm{~m}, x=0.148 \mathrm{~m}$ ) from $t=0.05 \mathrm{~s}$ to $t=0.15 \mathrm{~s}$ result shows that the magnitude of the velocity increases at the rear at about $1 / 3 b$ ( $b$ is the bottom span of the flowing granular materials) and reduces gradually to the flow front area as shown in Figs. 4.5 (b, c). The velocity profiles show a linear trend with little variations in magnitude at $x=0.148 \mathrm{~m}$. Fig. 4.6 illustrates the velocity evolution at $30^{\circ}$ inclination angle. The results indicate a similar trend as in the $45^{\circ}$ inclination slope to half of the 
flow depth with a sudden drop and later a linear increase to the free surface at $x=$ $0.15 \mathrm{~m}$. The velocity profiles may differ close to the free surface due to particles interaction in the dilute phase region. Both inclinations show similar phenomena for both physical and numerical studies at fixed position for different time showing the extent at which the flow is time dependent.
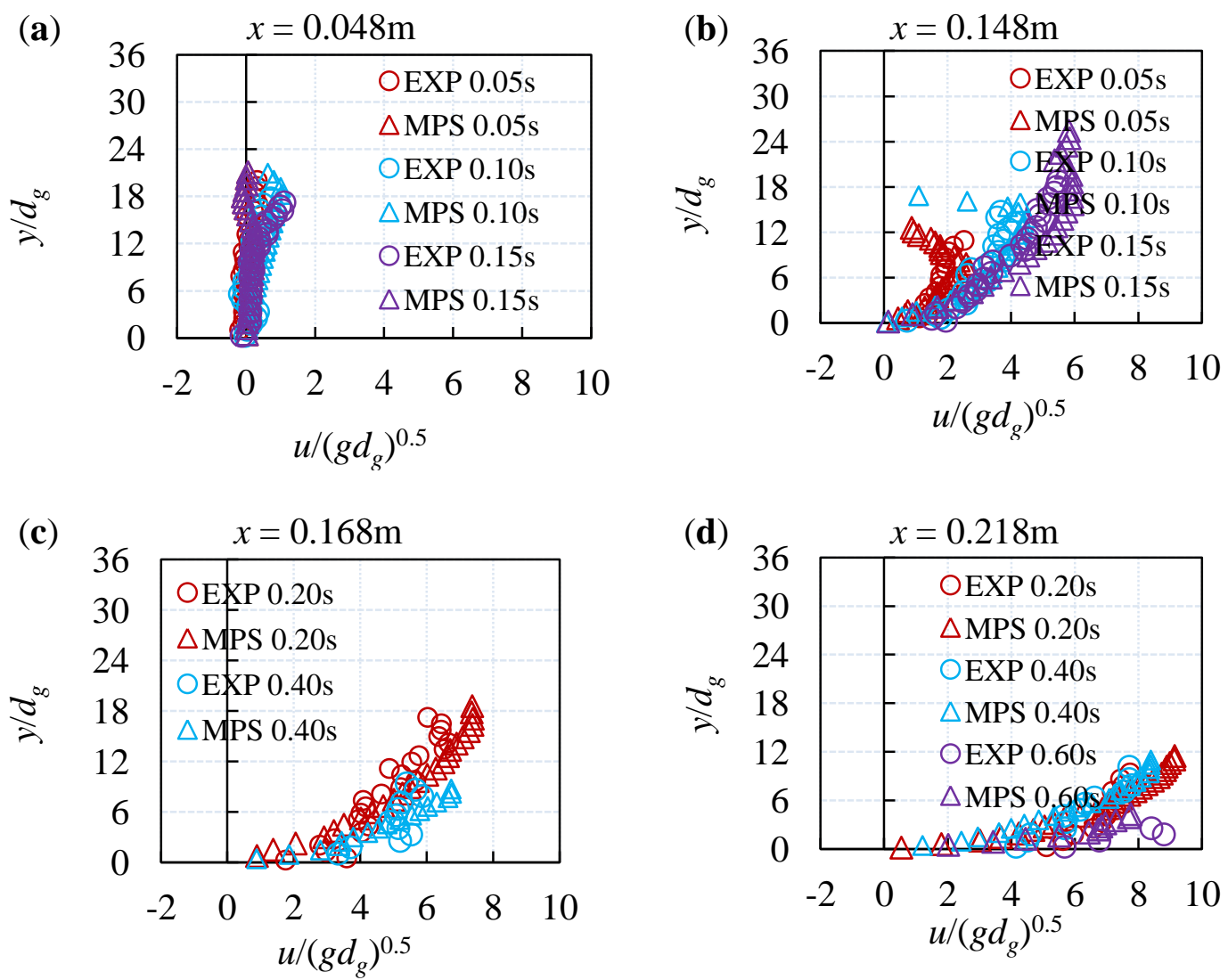

Fig. 4.5 Velocity evolution during acceleration down a slope of $45^{\circ}$ during initialisation when the granular materials start to deform at fixed sections. 

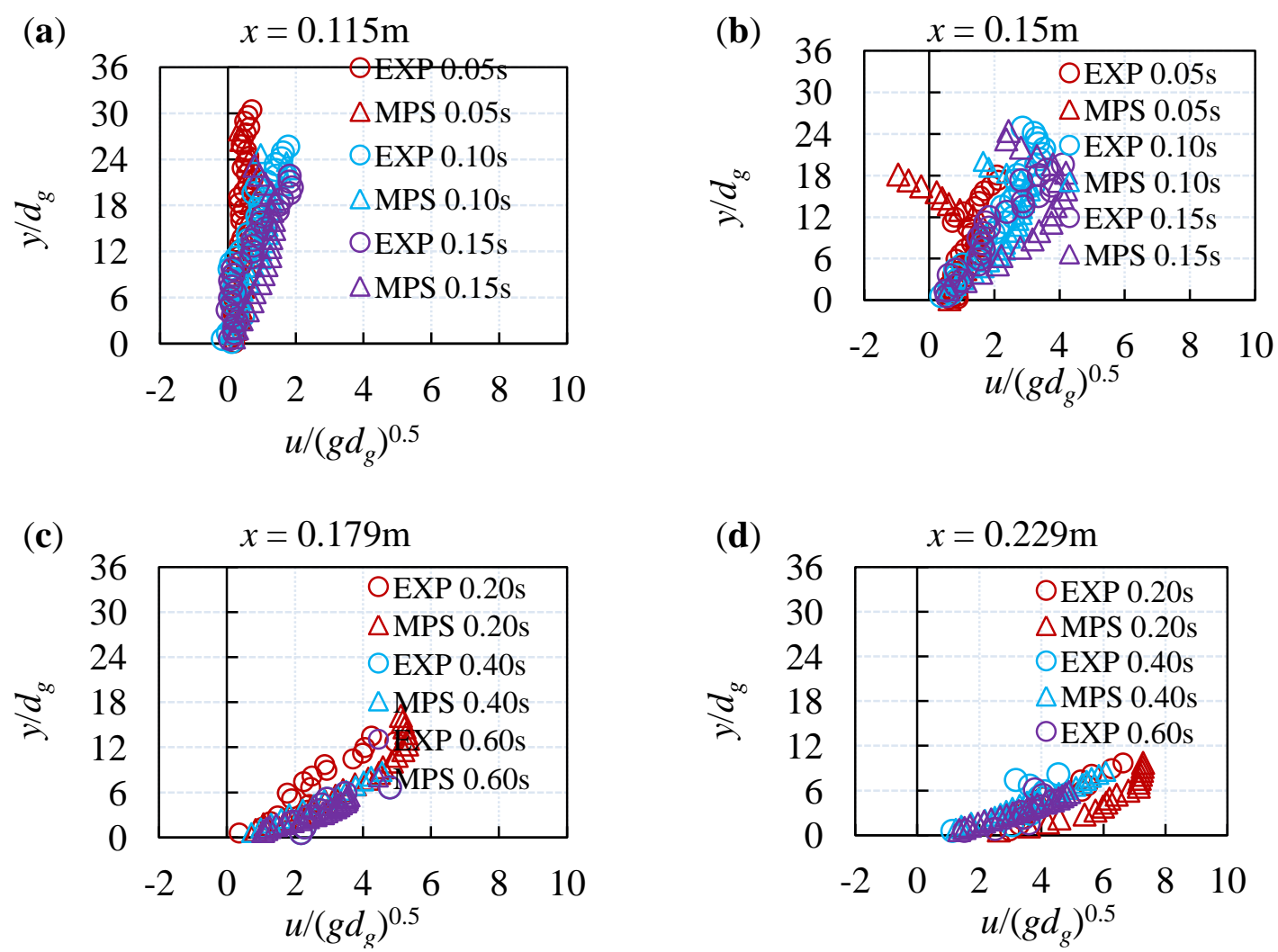

Fig. 4.6 Velocity evolution during acceleration down a slope of $30^{\circ}$ during initialisation when the granular materials start to deform at fixed sections. 


\subsection{Velocity contour}

The velocity contour shows the size and magnitude of the velocity distribution along the sheared granular materials from time $t=0.05 \mathrm{~s}$ to $t=0.4 \mathrm{~s}$. Fig. 4.7 illustrates the simulated and experimental velocity contour profile at $45^{\circ}$ inclination plane. The gradual decline in the size of the static region occurs until it attains minimal velocity at $t=0.4 \mathrm{~s}$. The maximum velocity is found at the frontal region with the least thickness while the minimum velocity is found at the rear portion close to the boundary. The rate of transformation from the distribution of velocity occurs very fast for steep inclinations. Although the granular materials continue to flow if not interrupted, the velocity distribution in the frontal top region is maximum while reducing gradually to the rear throughout the deformation of the granular materials. Fig. 4.8 shows that with an inclination angle of $30^{\circ}$ which has a larger static region as compared to $45^{\circ}$ but with the same similar velocity distribution phenomena. Although the thickness of the quasi-static phase reduces gradually during deformation, the bottom particles at this state maintain a minimal depth before dissipation due to increase in its velocity. The velocity distribution range is found to increase with time until its minimal depth is attained forming a uniform strip of velocity distribution. The numerical simulation showed good agreement to the experimental findings. Slight discrepancy can be observed at $t=0.10 \mathrm{~s}$ and $t=0.2 \mathrm{~s}$ where the particles dissipate from the static region in the simulated results. This observation is caused by the boundary and interface control between the sliding gate and the deforming granular material in the numerical studies. These deflected particles can be seen at the top free surface of the flowing granular materials which would later disappear at a later time step in the numerical study. These deflected particles were not used when estimating the results for the flow properties. 

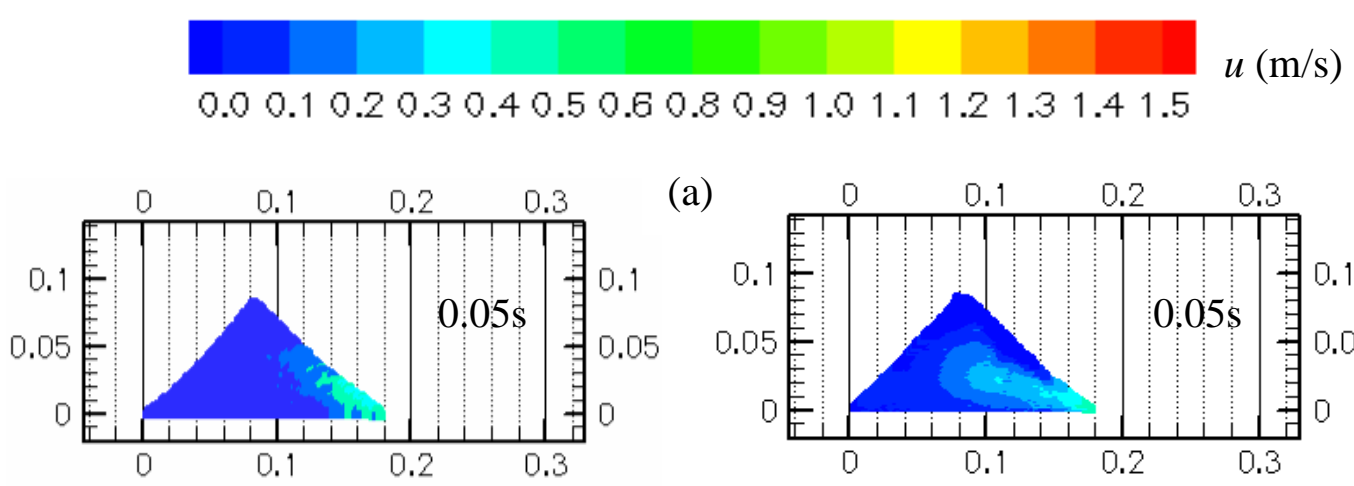

(a)

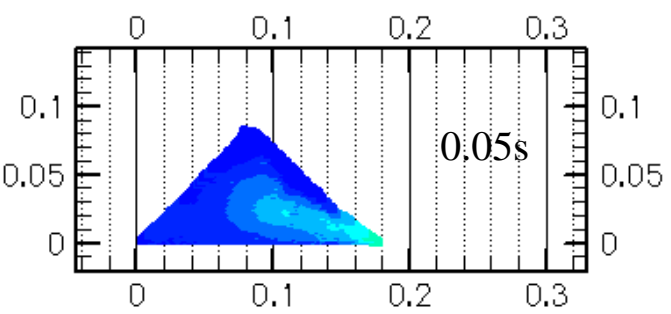

(b)
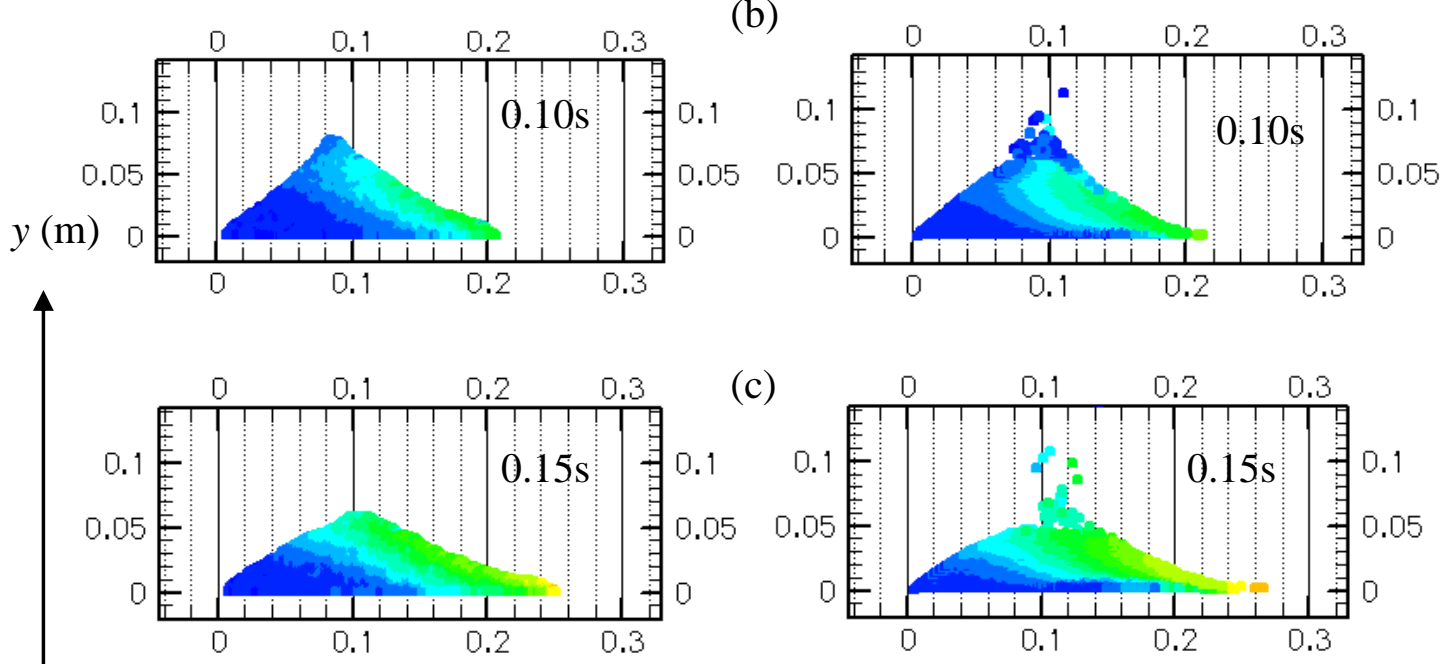

(c)

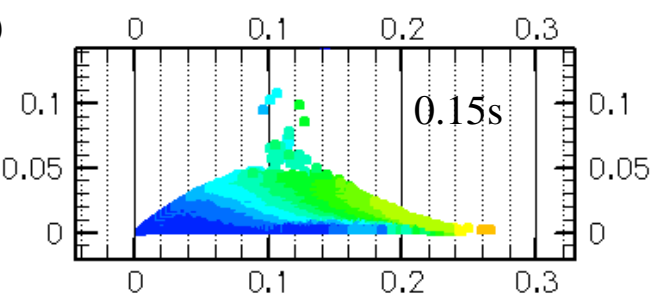

(d)
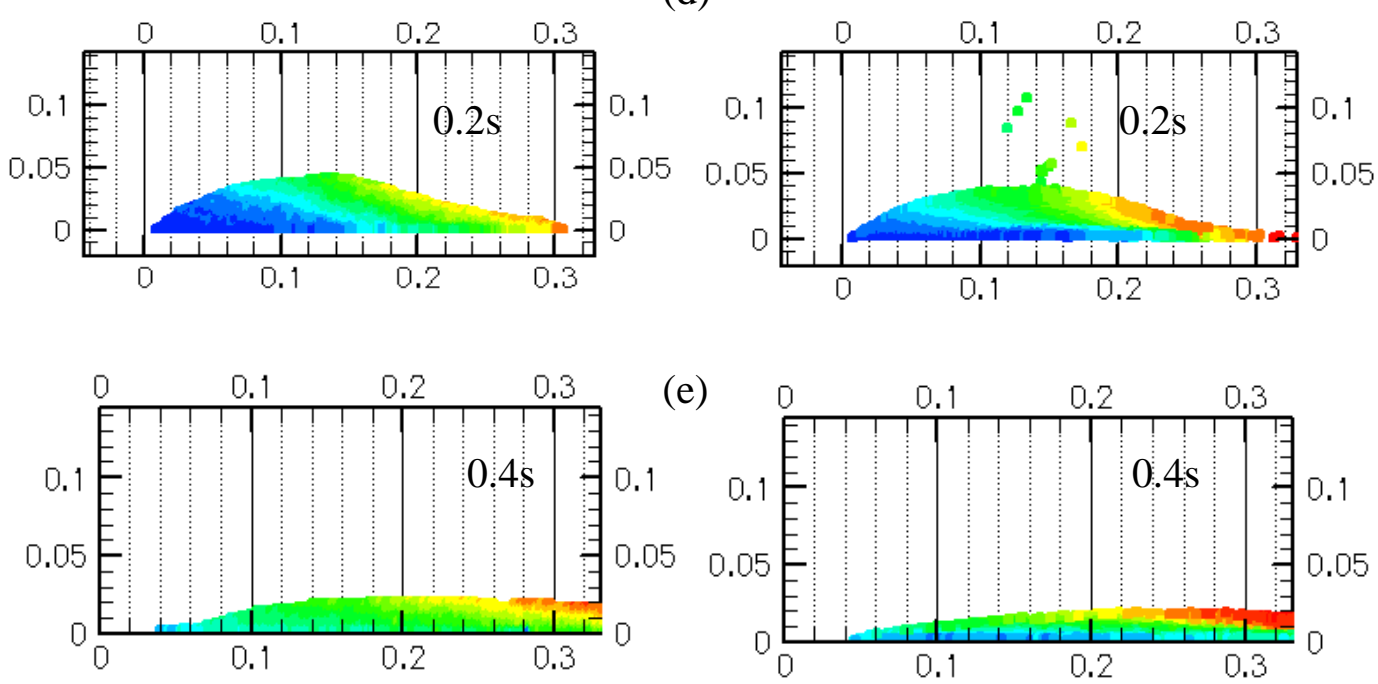

(e)

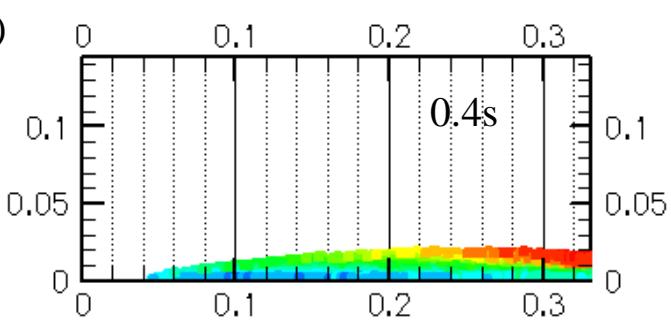

EXP MPS $x(\mathrm{~m})$

Fig. 4.7 Velocity contour plot profile for $45^{\circ}$ slope showing comparison between experimental and numerical results for time (a) $0.05 \mathrm{~s} \mathrm{(b)} 0.10 \mathrm{~s} \mathrm{(c)} 0.15 \mathrm{~s}$ (d) $0.2 \mathrm{~s}$ (e) $0.4 \mathrm{~s}$ 


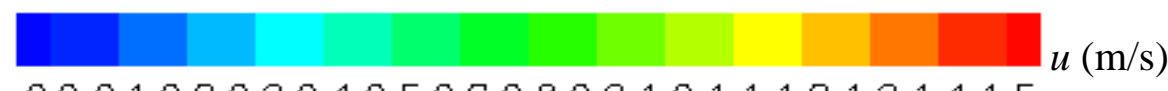
$0.0 \quad 0.10 .20 .30 .40 .50 .60 .80 .91 .01 .11 .21 .31 .41 .5$ $(\mathrm{m} / \mathrm{s})$

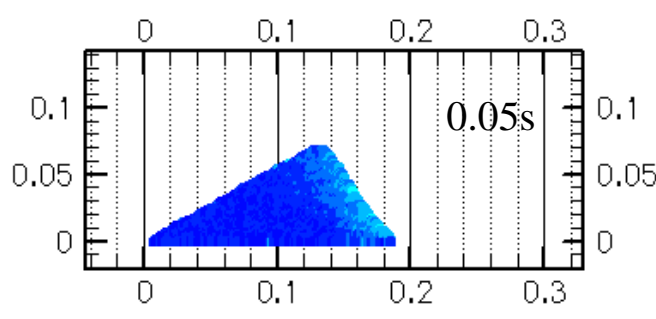

(a)
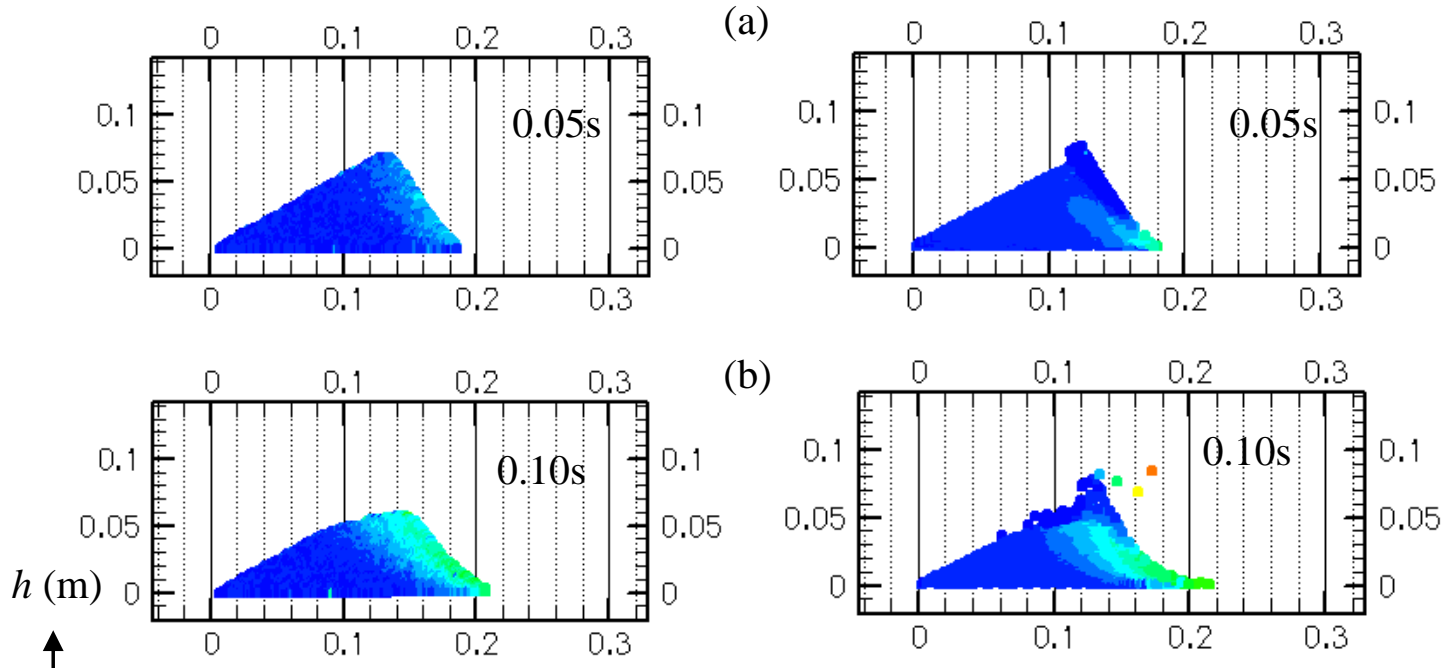

(b)
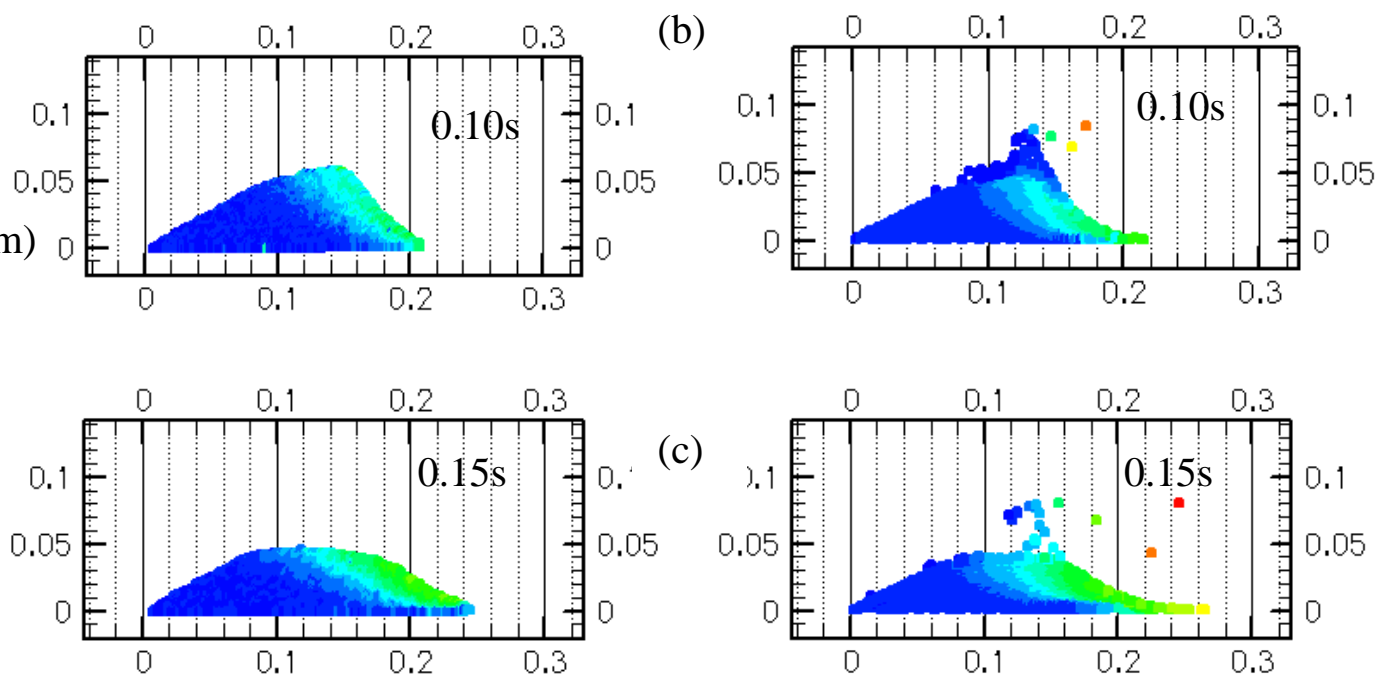

(c)
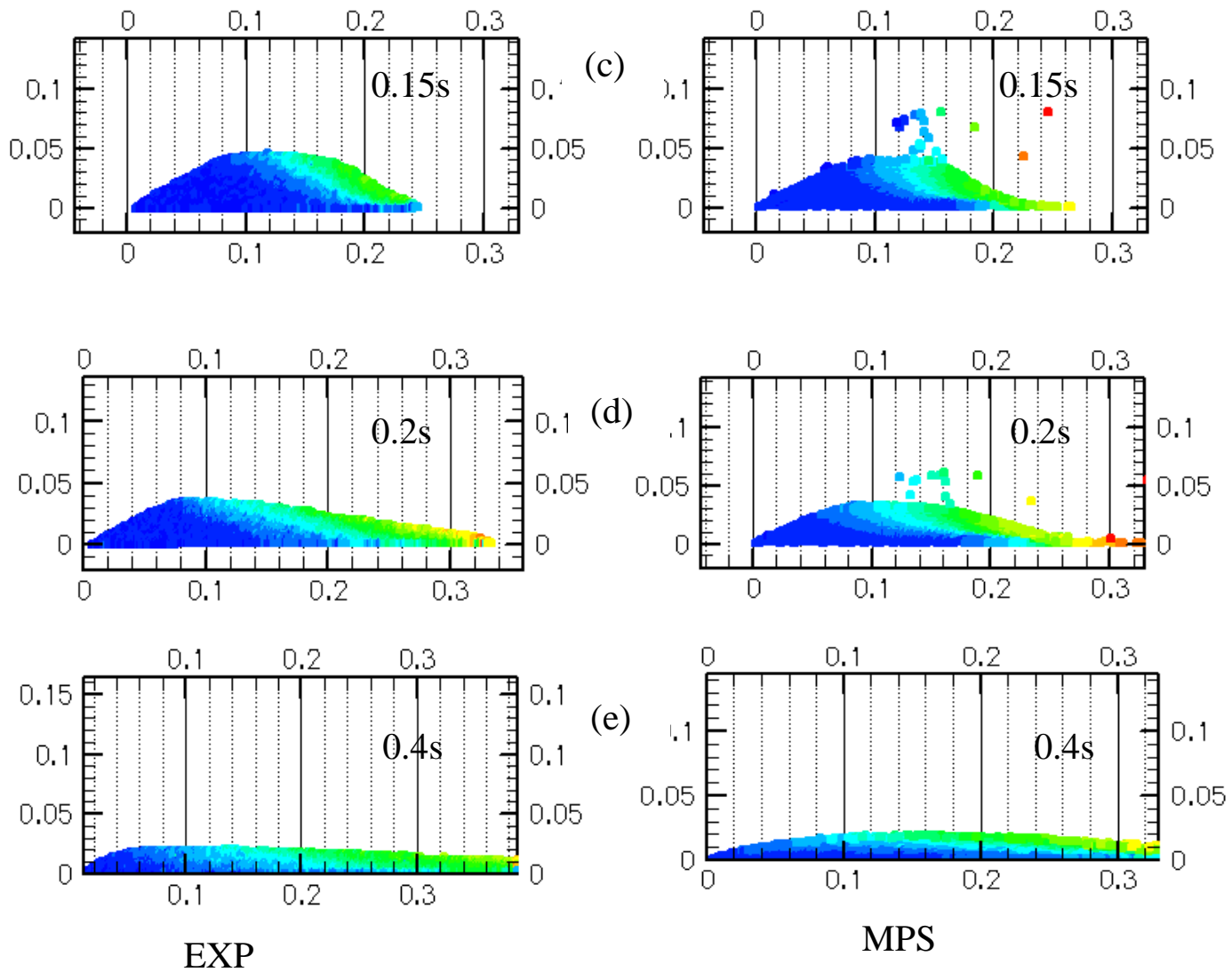

$x(\mathrm{~m})$

Fig. 4.8 Velocity contour plot profile for $30^{\circ}$ slope showing comparison between experimental and numerical results for time (a) $0.05 \mathrm{~s}$ (b) $0.10 \mathrm{~s} \mathrm{(c)} 0.15 \mathrm{~s}$ (d) $0.2 \mathrm{~s}$ (e) $0.4 \mathrm{~s}$ 


\subsection{Shear banding in unsteady states}

Granular deformation results from the localisation of strain formed during imbalance of forces of particles from initialisation. The frictional resistance from the interaction between particles leads to vortex formation instantaneously from the effect of gravitational force which exists from the quasi-static phase as shown in Figs. 4.9 and 4.10. The shear band is estimated by using the Froude number $\left(u / \sqrt{ } g d_{g}\right)$. The connection between vortices and shear band was stated to be from the linear combination of pure shear and particle rotation around the core regions (Crosta et al. 2015). The characterisation of these vortex cores determines the failure phenomena of the granular materials. The areas of failure form isolines at all substrate layers at an approximate angle relative to the channel boundary. These isolines correspond to layers with different Froude distribution influenced by gravitational force giving anisotropic behaviour, which results into attractive interaction as suggested by Charles Campbell (1989). The thickness of the granular materials between the isolines decreases with time and in height developing into multiple shears banding as the material deform. This nature of localisation is dependent on the transformation from the quasi-static state to the kinetic state. The behaviour of deformation of several materials differ, the rheology model produces the characteristics of the macroscopic constitutive description of the granular materials but failed to show instability phenomena during deformation. While studying granular flows on erodible and non-erodible inclines, Crosta et al. (2015) results showed a similar phenomenon on a $22^{\circ}$ slope. Before the arrest of the flow a stepped profile arises from localized shear bands which increase in number over time. The decrease in the static region transits into multiple shear bands during the collapse of the granular materials down the slope. 


\subsection{Shear}

The rheology from microscopic observation shows the shear stress, shear rate and coefficient of friction in the granular flow during slippage as shown in Figs. 4.11 (ad) for $45^{\circ}$ slope. It should be noted that shear is not measured but calculated based on the velocity gradient from the model results. The evolution of the shear stress shows a rapid increase from initialisation to a yielding point when the stress began to decrease over time after attaining its peak stress Fig. 4.11(a). The shear stress in the material had a sudden increase from initialisation point and subsequently reduced as shear rate increases. The increase in the shear rate as time increases led to reduction in the height of the flowing granular materials which was approximately steady at height $0.018 \mathrm{~m}$. At time $0.1 \mathrm{~s}$ the shear stress in the material gradually reduces as time increases giving the granular materials more resistivity to limit further segregation, hence the maintenance of the uniform minimum height of flow as the material propagates infinitely. The relative displacement of these particles at respective shear zone increases and causes dilation in the granular flow. This dilatancy leads to dispersion and transfer of shear zones as a result of resistance from neighbouring particles during collision. Several fluctuations as a result of dynamic equilibrium between the local contraction of particles and dilation during granular flow show negligible shear stress at the surface profiles. The properties of the granular materials exhibit frictional angles ranging from $\theta_{s}$ to $\theta_{2}$ which is relatively uniform as time and shear rate increases corresponding to a very loose material shown in Fig. 4.11(b). A solid to liquid behaviour is finally observed after $0.3 \mathrm{~s}$ with an approximate uniform friction coefficient, a process perfectly reversible as the rate of shear in the material is increased as shown in Fig. 4.11(c). The shear stress increased linearly at initialisation as the shear rate increases and later decreases while remaining constant as shown in 
Fig. 4.11(d). In general the granular flow exhibits a thixotropic behaviour observed from Figs. 4.11(a- d).
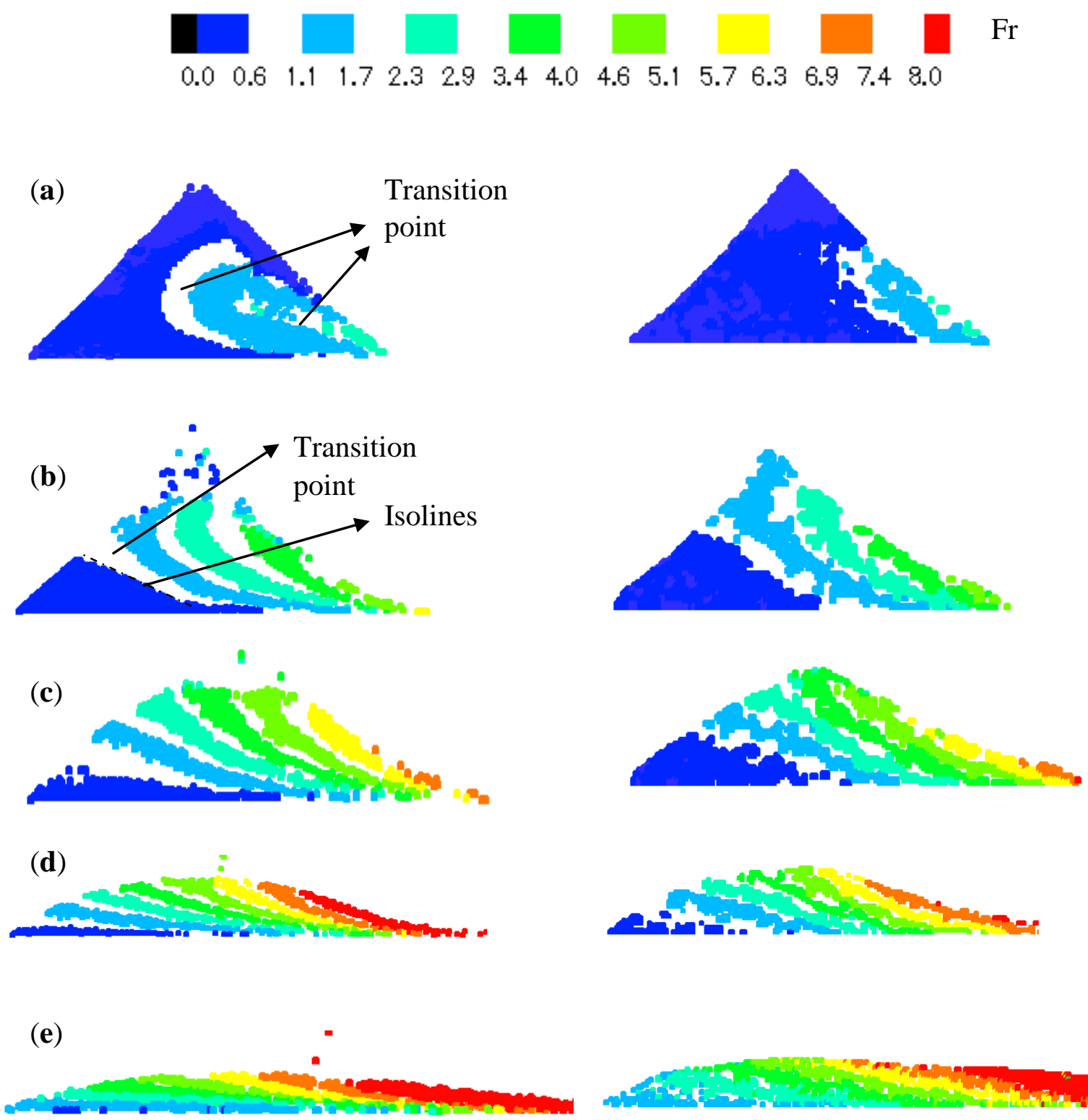

MPS

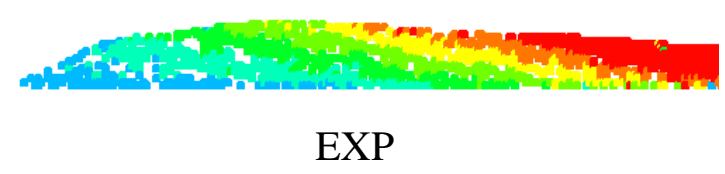

Fig. 4.9 Multi-Shear band layers for $45^{\circ}$ slope showing comparison between experimental and numerical results for time (a) $0.05 \mathrm{~s} \mathrm{(b)} 0.10 \mathrm{~s}$ (c) $0.15 \mathrm{~s} \mathrm{(d)} 0.20 \mathrm{~s}$ (e) $0.30 \mathrm{~s}$ 

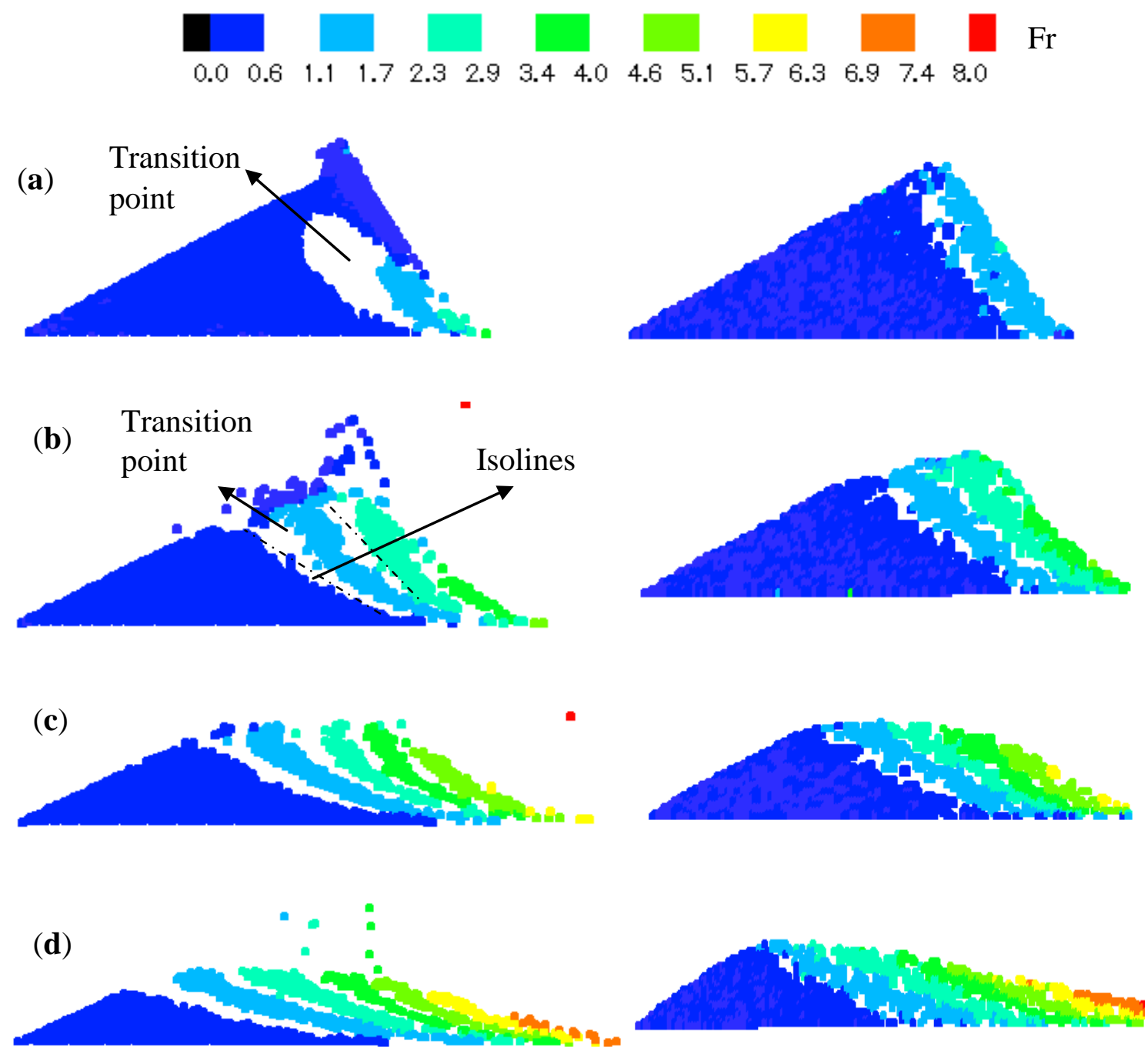

(e)

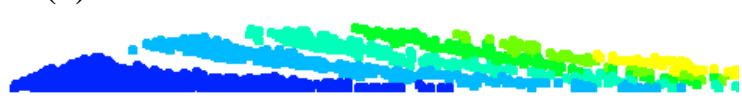

MPS

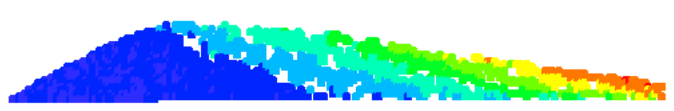

EXP

Fig. 4.10 Multi-Shear band layers for $30^{\circ}$ slope showing comparison between experimental and numerical results for time (a) $0.05 \mathrm{~s}$ (b) $0.10 \mathrm{~s}$ (c) $0.15 \mathrm{~s} \mathrm{(d)} 0.20 \mathrm{~s}$ (e) $0.30 \mathrm{~s}$ 


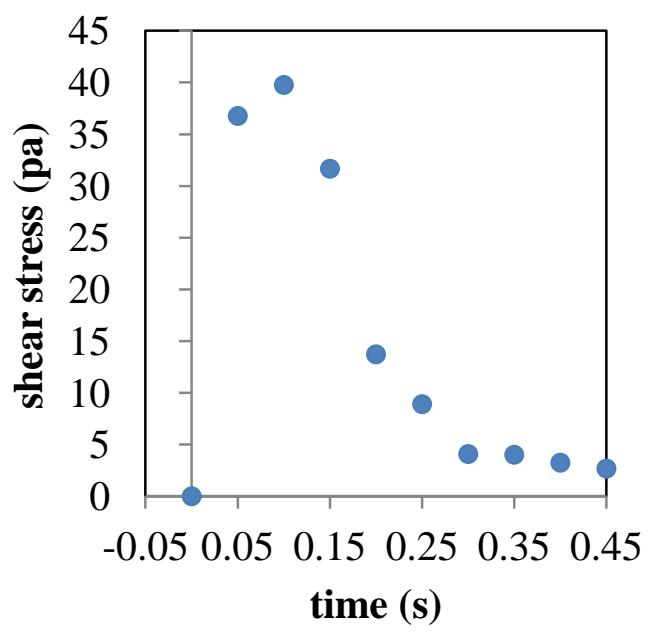

(a)

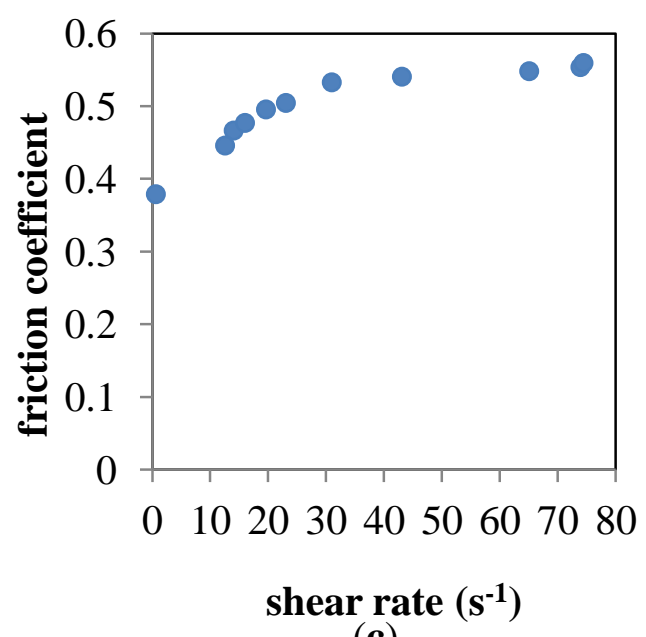

(c)

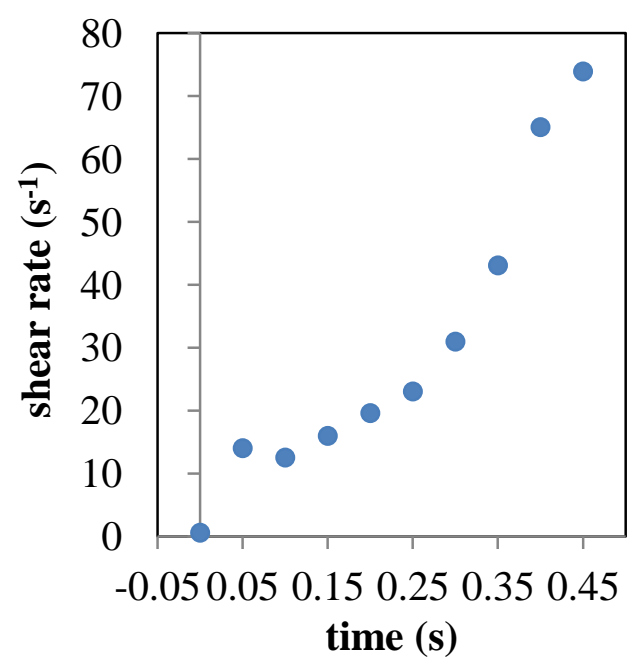

(b)

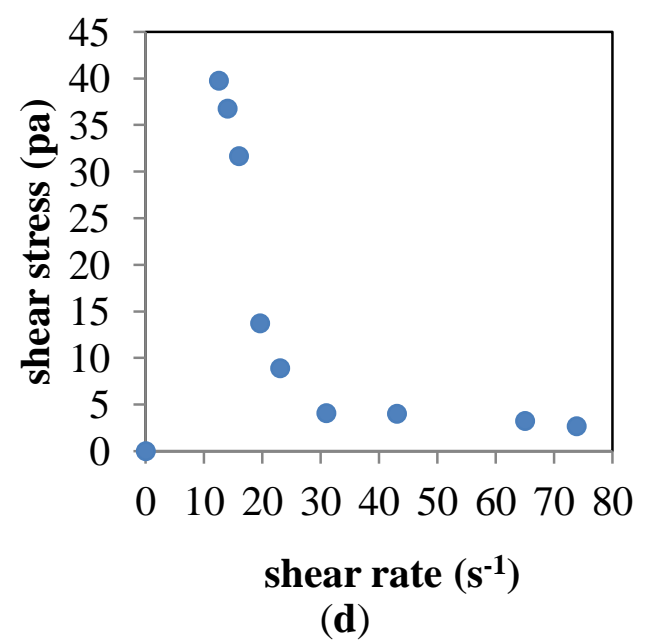

(d)

Fig. 4.11 Rheological behaviour of the granular materials where (a) and (b) shows shear stress and shear rate as a function of time and (c) to (d) shows the friction coefficient and shear stress as a function of shear rate

\subsection{Shear distribution}

The distribution of shear stress shows the yielding stress behaviour of the granular materials. During the quasi-static regime, the granular materials deforms slowly with the high magnitude of the shear stress found at half left and the least at the free surface of the granular materials as shown in Fig. 4.12(a). This shows that the particles close to the free surface are in the kinetic mode where individual particles 
possess the momentum of their motion while in the frictional and collisional mode, momentum is transferred from particle to particle interactions Campbell, 1989. This region is mostly the dense region of the bulk granular materials. The shear stress in the granular materials at the front wave region is minimal with slippage from the front bottom region having maximum shear rate as shown in Fig. 4.12(b). As the effect of the shear stress reduces, the shear rate increases at the boundary of the granular materials.

\subsection{Surface profile}

The free surface dynamics is dependent on the rate of flow and the thickness $h(t)$ of the profile related to the time evolution of internal stresses of the granular materials. Having found good agreement between physical and the numerical results, the increase in the inclination from $30^{\circ}$ to $45^{\circ}$ led to higher acceleration but yet the result showed that from $0 \mathrm{~s}$ to $0.10 \mathrm{~s}$, the rate of deformation of the material with respect to the material thickness $h(t)$ for $45^{\circ}$ was slower compared to the $30^{\circ}$ as shown in Figs. 4.13 and 4.14. It was observed that the rate of reduction at the peak of the granular materials increases after the deformed material approaches the angle of repose on the inclined plane for $30^{\circ}$ as opposed to the expectation of assuming that granular materials on the $45^{\circ}$ would flow faster from initialisation. This might be due to the differences in the setup and initial granular material profile when at rest.

The rear free surface of the flowing mass at $0.5 \mathrm{~s}$ to $0.2 \mathrm{~s}$ maintained acceptable positions asides the top of the free surface and the frontal region of the flowing mass as shown in Fig. 4.13. The depth of the flow for the simulation in $45^{\circ}$ is slightly underestimated unlike that of the $30^{\circ}$ as shown in Fig. 4.14. 
$0.0 \quad 7.1 \quad 14.321 .428 .635 .742 .950 .057 .164 .371 .478 .685 .792 .9100 .0 \quad \gamma\left(\mathrm{s}^{-1}\right)$
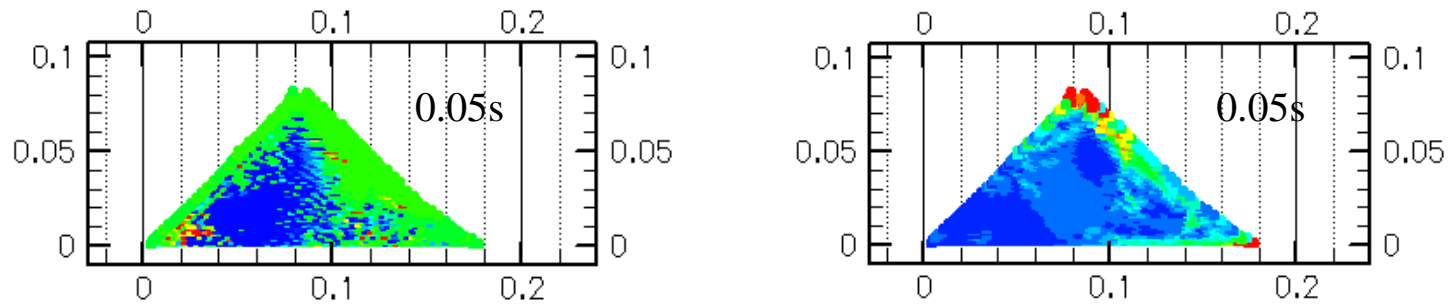

$y(\mathrm{~m})$
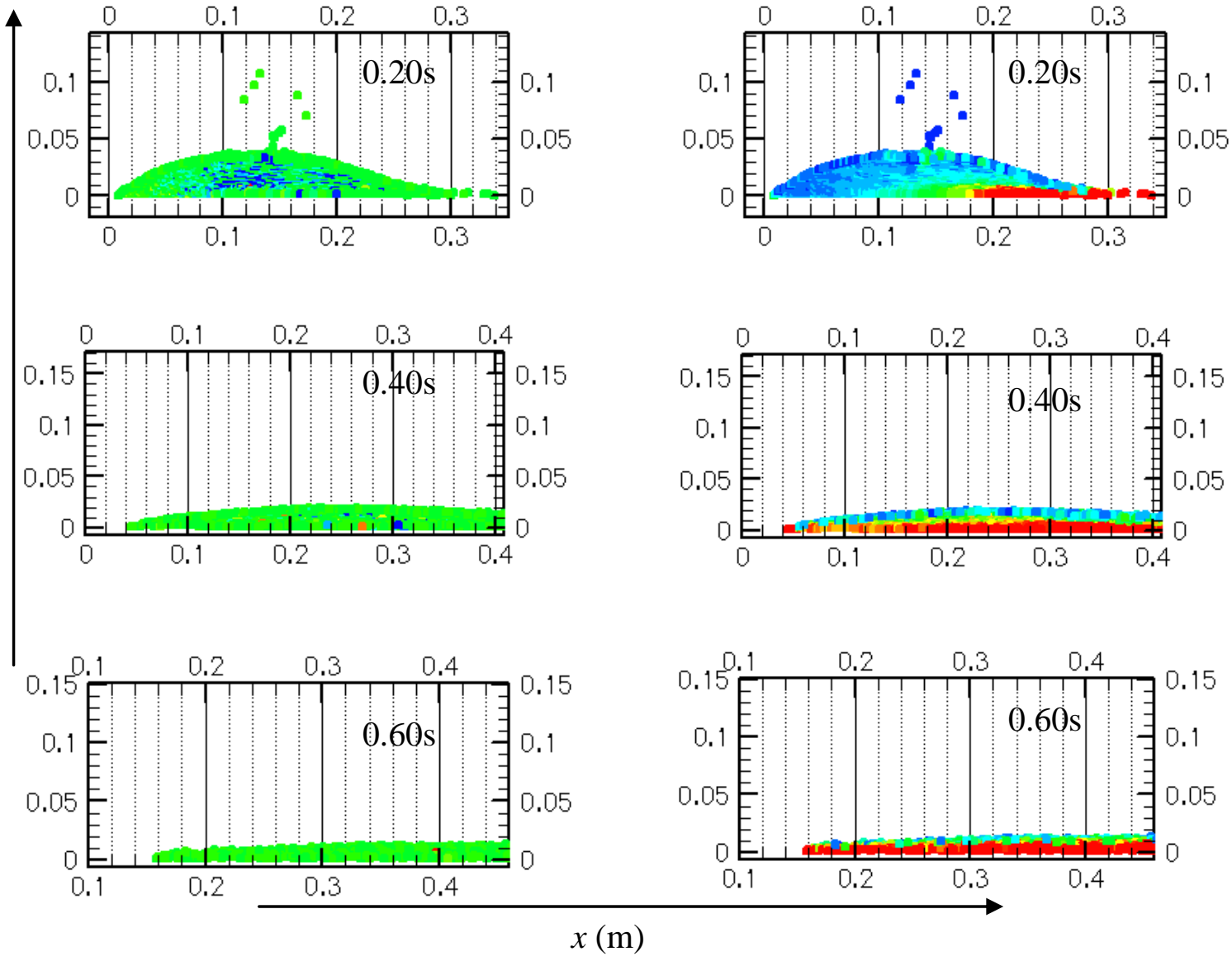

(a) Shear stress

(b) Shear rate

Fig. 4.12 Shear stress and shear rate for $45^{\circ}$ slope. 
(a)

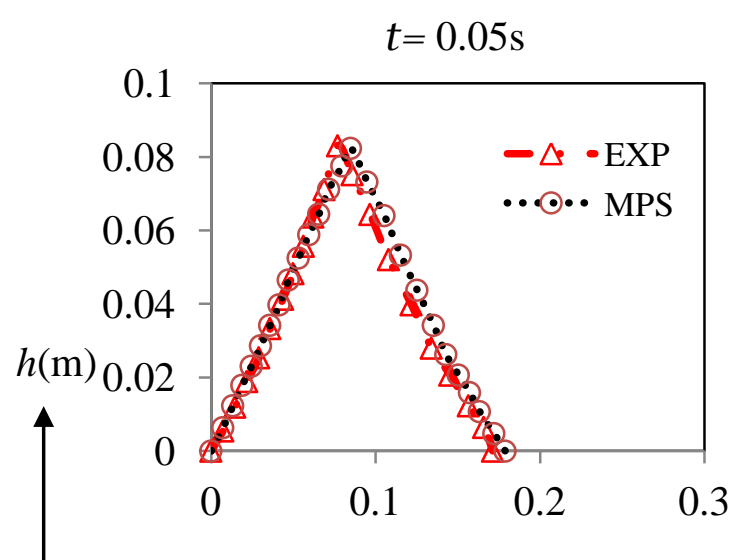

(c)

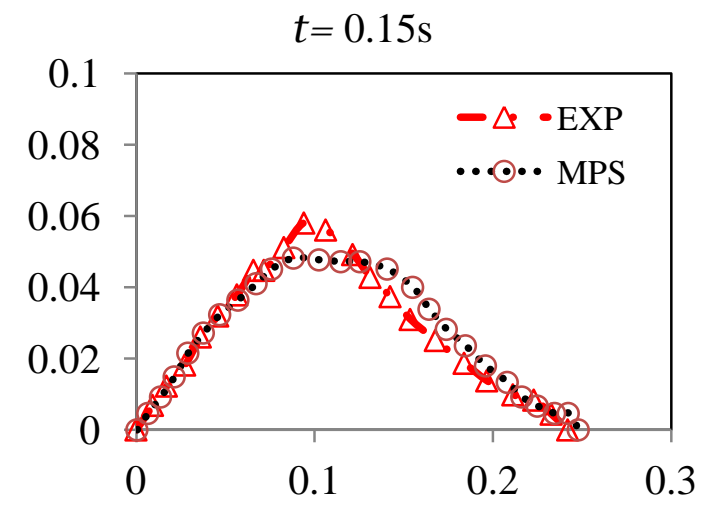

(b)

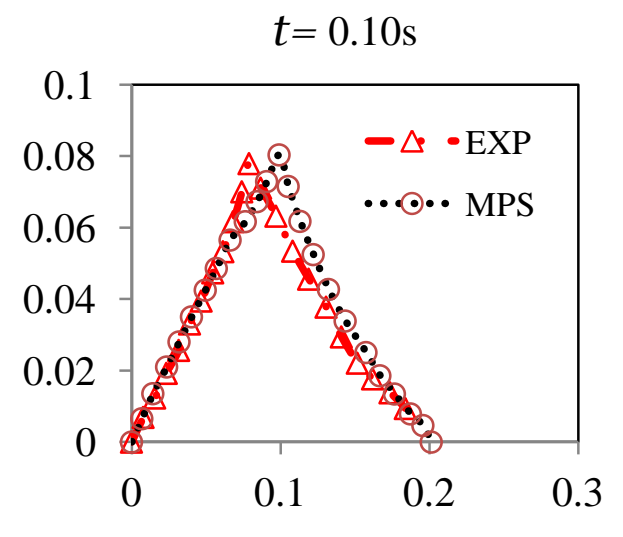

(d)

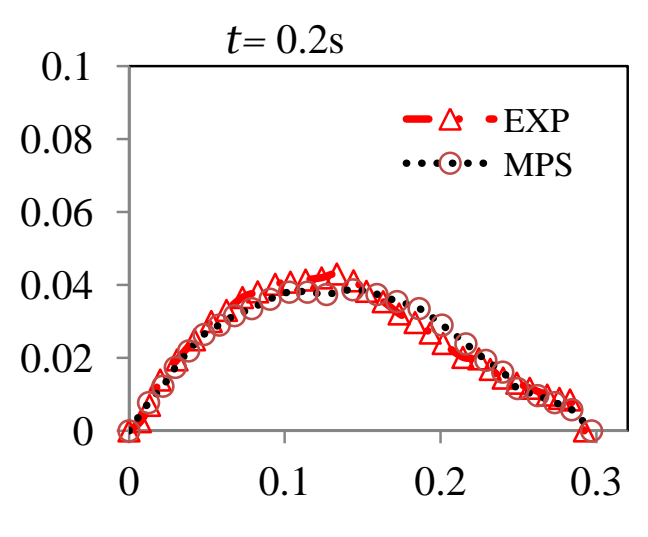

$x(\mathrm{~m})$

Fig. 4.13: Surface profile for $45^{\circ}$ slope showing comparison between experimental and numerical results for time (a) $0.05 \mathrm{~s}$ (b) $0.10 \mathrm{~s}$ (c) $0.15 \mathrm{~s} \mathrm{(d)} 0.2 \mathrm{~s}$ 
(a)

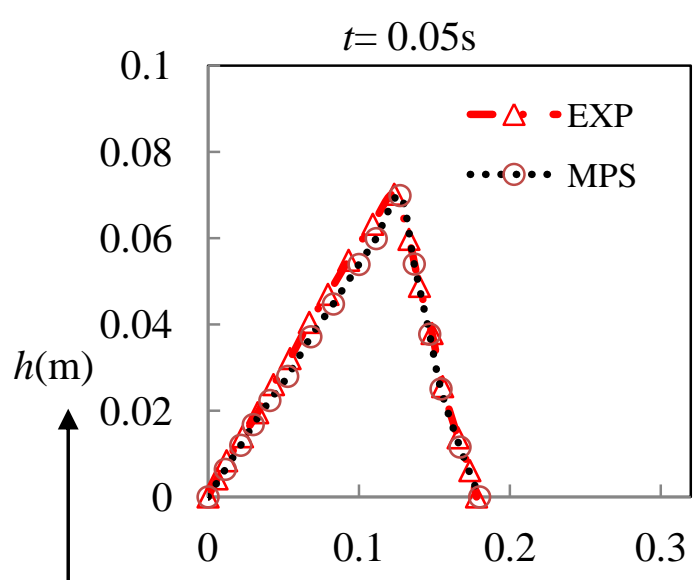

(c)

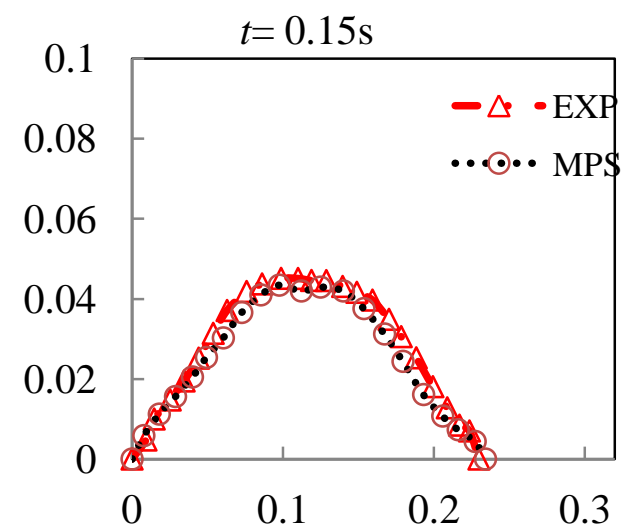

(b)

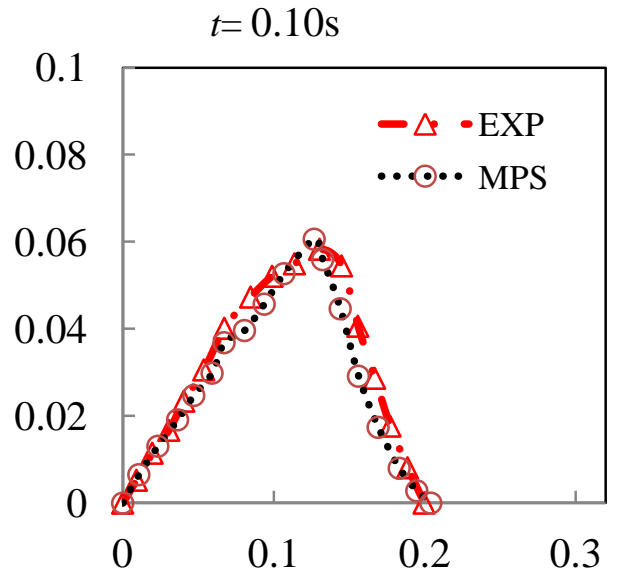

(d)

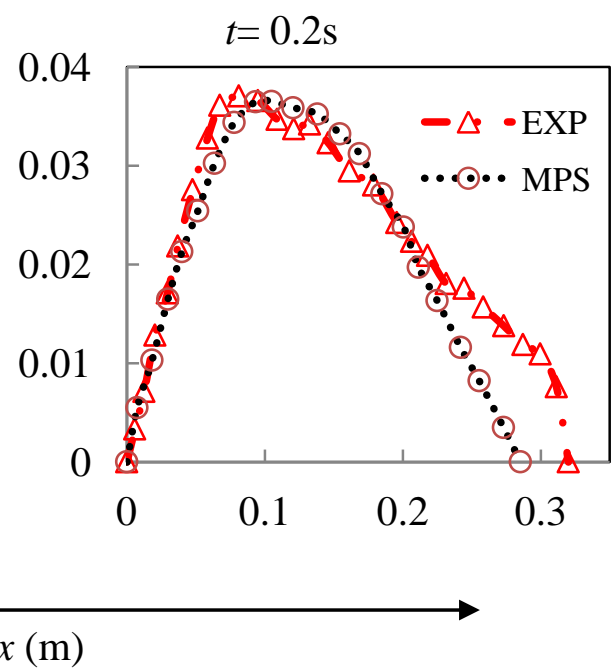

Fig. 4.14 Surface profile for $30^{\circ}$ slope showing comparison between experimental and numerical results for time (a) $0.05 \mathrm{~s}$ (b) $0.10 \mathrm{~s} \mathrm{(c)} 0.15 \mathrm{~s} \mathrm{(d)} 0.2 \mathrm{~s}$ 
At $0.2 \mathrm{~s}$ a sudden increase in the rate of shear at the wave front was observed for the physical study. The frontal positions of granular particles increased with an average of $3 \%$ from 0 s to $0.2 \mathrm{~s}$ in both studies.

\subsection{Wave front}

The wave front from the propagation of the material occurs at the frontal region of the material when the depth of the granular materials is approximately zero $(h \approx 0)$. Particle dispersion are forced towards areas of least shear which also is a limitation in the use of the rheology model, especially having that the flow front is unsteady with segregation of particles with higher velocities (Xu and Jin 2016). Particles at the wave front have maximum velocities. The length of the inclined channel limits the experimental results to four time steps with 0.05 increments $(0.05 \mathrm{~s}-0.20 \mathrm{~s})$ while the numerical shows from $(0.05 \mathrm{~s}$ to $0.4 \mathrm{~s})$ as shown in Fig. 4.15. The results are plotted and compared for both $45^{\circ}$ and $30^{\circ}$ giving acceptable prediction. Unlike arresting granular flows on slopes where existing mathematical formulations can be used to predict the flow front, run-out and arrest time, continuous flowing granular materials on inclined plane have no existing formulations to predict the flow front as the runout and arrest time does not exist for such flows. It can be observed from Fig. 4.15 that the difference in slope between $45^{\circ}$ and $30^{\circ}$ show similar flow front at the early stages of the granular flow similar to observations observed by Lee and Yang 2016 for arresting flows at the earliest stages for different slopes. 


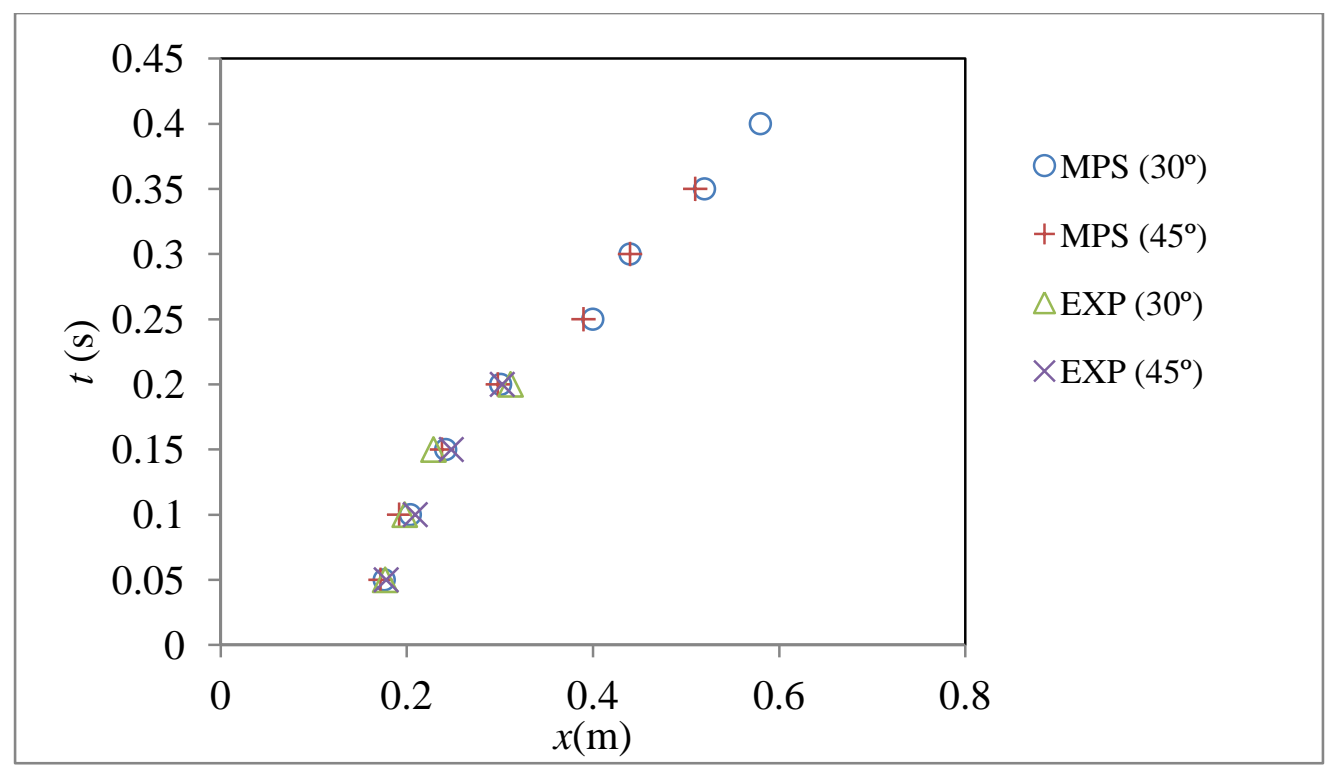

Fig. 4.15 Wave front time evolution for $45^{\circ}$ and $30^{\circ}$ slope showing comparison between experimental and numerical results

\subsection{General Discussion}

The approach used in this study is able to show acceptable results with physical observations for unsteady granular flow for both microscopic and macroscopic properties. Although the model did not capture the granular behaviour at the frictional phase, measured properties were similar to observations from the physical study. This limitation from the frictional rheological model may be from the friction and dilatancy law where the sheared granular flow is controlled by the inertia number which is relatively small when the flow is initiated. 


\section{CHAPTER 5: CONCLUSION}

\subsection{Summary}

In this thesis, we have presented experimental and numerical studies on the rheological properties of granular collapse on inclined plane. These rheological properties deal with complexities in the granular fluids, constituting the need to properly understand granular matter. The introduction of the empirical $\mu(I)$ rheology model into MPS as a coupled set is used to describe the properties of the granular behavior for steady and unsteady flows. This granular behavior is governed by the contact dynamics between particles at the dense state where momentum transport is developed from inter-particle collision and the effect of gravity. A major part of this thesis is concerned with the velocity distribution and effect of gravity considered in its interrelation with the formation of shear bands. The two inclination angles discussed in this thesis show no major differences in the deformation of the granular flow properties. Although the initial geometry of the static granular material slightly differs, it converges after a period of time to a similar flow dynamics for different inclination angles. This indicates that granular collapses under the same configuration are governed by similar constitutive law.

The rate of deformation and transition of the dense granular materials affects the velocity distribution at the inflection points between the quasi-static and kinetic phase resulting into instabilities. Despite these instabilities a linear relationship exists for the velocity at several transverse sections. At fixed transverse locations, the velocity profile is observed to maintain a linear relationship between dimensionless velocity $\stackrel{x}{u}=u / \sqrt{ } g d_{g}$ and position $\stackrel{x}{y}=y / d_{g}$ at different time. The selections of different fixed positions limit the effect of instability in the granular flow properties when 
considering the changes in time during the deformation of the granular materials. Constant changes in the depth of unsteady granular flows over time makes it difficult to give similar flow pattern observed in steady flow, where there exist relationship between the height and velocity of the granular flow.

The functional rheological parameters used in the simulation of the granular collapse captured some of the complex behavior at larger values of the inertia number $I$, after the shear stress yields as the shear rate increases at the early stages of the granular collapse. Although the high value of $I$ contributes to particle dispersion at the flow front, the effect when compared to the experimental results is observed to be negligible. The deformation of the granular materials was observed to develop multiple shear bands linked by isolines at substrate layers giving anisotropic behaviour. The instantaneous effect of gravity initiates the frictional resistance from the cohesive interactionbetween particles resulting into the shear band formation. The localisation of strain is subject to the lack of force balance in the granular materials during deformation. Although there seems to be a relationship between gravity and viscous stresses, it is uncertain if this behavior is associated with the development of shear bands. The thickness of the shear bands increases and later diminishes as the depth of the granular materials tends to its minimum. The evolution of the shear stress increases linearly and reduces after attaining its peak stress. The distribution of the shear stress is found at the dense state of the granular materials. The free surface dynamics depends on the thickness of the granular profile and inclination angle related to the time evolution of internal stresses in the granular materials. Early stages of the granular flow are found to exhibit similar flow front for different slopes which might slightly differ at a later state. 
The coupled model used in this study is capable of capturing some complex behaviour and flow dynamics of the granular materials, suggesting that further empirical formulations can be used to enhance the friction laws of other configurations. Although the unsteady state of the granular flow still lacks theoretical description, the results presented in this thesis would aid in its formulation. The results from the coupled model show good agreement with experimental investigations recommending the method as a powerful simulation technique for granular avalanche. 


\section{References}

1. Ancey, Christophe.: Dry granular flows down an inclined channel: Experimental investigations on the frictional-collisional regime. Physical Review E 65.1011304 (2001)

2. Ancey, C., Coussot, P., \& Evesque, P.: A theoretical framework for granular suspensions in a steady simple shear flow. Journal of Rheology, 43(6), 16731699. (1999)

3. Ataie-Ashtiani, B., \& Farhadi, L.: A stable moving-particle semi-implicit method for free surface flows. Fluid dynamics research, 38(4), 241 (2006).

4. Azanza, Emmanuel, François Chevoir, and Pascal Moucheront.: Experimental study of collisional granular flows down an inclined plane. Journal of Fluid Mechanics 400, 199-227 (1999)

5. Bagnold, Ralph A.: Experiments on a gravity-free dispersion of large solid spheres in a Newtonian fluid under shear. Proceedings of the Royal Society of London. Series A. Mathematical and Physical Sciences 225, 1160, 49-63 (1954)

6. Barker, Thomas, D.G. Schaeffer, Michael Shearer, and J. M. N. T. Gray.: Well-posed continuum equations for granular flow with compressibility and $\mu(I)$-rheology. Proc. R. Soc. A 473, 220120160846 (2017)

7. Barker, Thomas, Schaeffer, D.G., Bohorquez, P. and Gray, J.M.N.T.: Wellposed and ill-posed behaviour of the $\mu(I)$-rheology model for granular flow. Journal of Fluid Mechanics 779, 794-818 (2015)

8. Berzi, D., C. G. Di Prisco, and D. Vescovi.: Constitutive relations for steady, dense granular flows. Physical Review E 84.3, 031301 (2011) 
9. Campbell, C. S.: The stress tensor for simple shear flows of a granular material. Journal of Fluid Mechanics 203, 449-473 (1989)

10. Chambon, Guillaume, Bouvarel, R., Laigle, D., \& Naaim, M.: Numerical simulations of granular free-surface flows using smoothed particle hydrodynamics. Journal of Non-Newtonian Fluid Mechanics 166, 12-13 698$712(2011)$

11. Chauchat, Julien, and Marc Médale.: A three-dimensional numerical model for dense granular flows based on the $\mu(\mathrm{I})$ rheology. Journal of Computational Physics 256, 696-712 (2014)

12. Crosta, G. B., Imposimato, S., \& Roddeman, D.: Granular flows on erodible and non erodible inclines. Granular Matter, 17(5), 667-685 (2015)

13. Cui, Xinjun, and J. M. N. T. Gray.: Gravity-driven granular free-surface flow around a circular cylinder. Journal of Fluid Mechanics 720, 314-337 (2013)

14. Da Cruz, Frédéric, et al.: Rheophysics of dense granular materials: Discrete simulation of plane shear flows. Physical Review E 72.2, 021309 (2005)

15. Dunatunga, Sachith, and Ken Kamrin.: Continuum modelling and simulation of granular flows through their many phases. Journal of Fluid Mechanics 779, $483-513(2015)$

16. Ertaş, D., Grest, G. S., Halsey, T. C., Levine, D., \& Silbert, L. E.: Gravitydriven dense granular flows. EPL (Europhysics Letters), 56(2), 214 (2001)

17. Fan, Y., Umbanhowar, P. B., Ottino, J. M., \& Lueptow, R. M.: Kinematics of monodisperse and bidisperse granular flows in quasi-two-dimensional bounded heaps. Proc. R. Soc. A, 469, (2157), 20130235 (2013) 
18. Fei, Minglong, Qicheng Sun, Deyu Zhong, and Gordon GD Zhou.: Simulations of granular flow along an inclined plane using the Savage-Hutter model. Particuology 10.2, 236-241 (2012)

19. Forterre, Y., \& Pouliquen, O. Long-surface-wave instability in dense granular flows. Journal of Fluid Mechanics 486, 21-50 (2003)

20. Forterre, Yoël, and Olivier Pouliquen.: Flows of dense granular media. Annu. Rev. Fluid Mech. 40, 1-24 (2008)

21. Forterre, Yoël, and Olivier Pouliquen.: Longitudinal vortices in granular flows. Physical Review Letters 86.265886 (2001)

22. Frigaard, I.A. \& Nouar, C.: On the Usage of Viscosity Regularisation Methods for Visco-Plastic Fluid Flow. Computation. J. Non-Newtonian Fluid Mech. 127. 1-26. 10.1016/j.jnnfm.2005.01.003 (2005)

23. Gingold, Robert A., and Joseph J. Monaghan.: Smoothed particle hydrodynamics: theory and application to non-spherical stars. Monthly notices of the royal astronomical society $\mathbf{1 8 1 . 3} 375-389$ (1977)

24. Goujon, Celine \& Thomas, N \& Dalloz-Dubrujeaud, Blanche.: Monodisperse Dry Granular Flows on Inclined Planes: Role of Roughness. The European physical journal. E, Soft matter. 11. 147-57. 10.1140/epje/i2003-10012-0 (2003).

25. Ikari, Hiroyuki, and Hitoshi Gotoh.: SPH-based simulation of granular collapse on an inclined bed. Mechanics Research Communications 73, 12-18 (2016)

26. Ionescu, Ioan R., Anne Mangeney, François Bouchut, and Olivier Roche.: Viscoplastic modeling of granular column collapse with pressure-dependent rheology. Journal of Non-Newtonian Fluid Mechanics 219, 1-18 (2015) 
27. Jafari-Nodoushan, Ehsan, Ahmad Shakibaeinia, and Khosrow Hosseini.: A multiphase mesh-free method for continuum-based modeling of dry and submerged granular flows. arXiv preprint arXiv: 1708.04521 (2017)

28. Jessop, D., Hogg, A., Gilbertson, M., \& Schoof, C.: Steady and unsteady fluidised granular flows down slopes. Journal of Fluid Mechanics, 827, 67120. doi:10.1017/jfm.2017.458 (2017)

29. Jop, Pierre, Yoël Forterre, and Olivier Pouliquen.: A constitutive law for dense granular flows. Nature, 441, (7094), 727 (2006)

30. Jop, Pierre, Yoël Forterre, and Olivier Pouliquen.: Crucial role of sidewalls in granular surface flows: consequences for the rheology. Journal of Fluid Mechanics 541, 167-192 (2005)

31. Jop, Pierre.: Rheological properties of dense granular flows. Comptes rendus physique 16, 1 62-72 (2015)

32. Koshizuka, S., Nobe, A., \& Oka, Y.: Numerical analysis of breaking waves using the moving particle semi-implicit method. International Journal for Numerical Methods in Fluids, 26(7), 751-769 (1998)

33. Koshizuka, Seiichi, and Yoshiaki Oka.: Moving-particle semi-implicit method for fragmentation of incompressible fluid. Nuclear science and engineering 123.3, 421-434 (1996)

34. Kumaran, V.: The constitutive relation for the granular flow of rough particles, and its application to the flow down an inclined plane. Journal of Fluid Mechanics 561, 1 - 42 (2006)

35. Kyung Sung Kim, Moo Hyun Kim, and Jong-Chun Park.: Development of Moving Particle Simulation Method for Multiliquid-Layer Sloshing. 
Mathematical Problems in Engineering, vol., Article ID 350165, 13 pages, doi:10.1155/2014/350165 (2014)

36. Lagrée, P-Y., Lydie Staron, and Stéphane Popinet.: The granular column collapse as a continuum: validity of a two-dimensional Navier-Stokes model with a $\mu(I)$-rheology. Journal of Fluid Mechanics 686, 378-408 (2011)

37. Lee, K. L., \& Yang, F. L.: Asymptotic analysis for the propagation and arresting process of a finite dry granular mass down a rough incline. Journal of Fluid Mechanics 806, 234-253 (2016)

38. Lo, C. Y., M. D. Bolton, and Y. P. Cheng.: Velocity fields of granular flows down a rough incline: a DEM investigation. Granular Matter 12.5, 477-482 (2010)

39. Lucy, Leon B.: A numerical approach to the testing of the fission hypothesis. The astronomical journal 82 1013-1024 (1977)

40. Lueptow, R. M., Akonur, A., \& Shinbrot, T.: PIV for granular flows. Experiments in Fluids, 28(2), 183-186. (2000)

41. MiDi, G. D. R.: On dense granular flows. The European Physical Journal E 14.4, 341-365 (2004)

42. Mollon, G. A.: Multibody meshfree strategy for the simulation of highly deformable granular materials. International Journal for Numerical Methods in Engineering, 108 (12), 1477-1497 (2016)

43. Monaghan, J. J.: Simulating free surface flows with SPH. Journal of computational physics, 110(2), 399-406 (1994)

44. Pouliquen, Olivier.: Scaling laws in granular flows down rough inclined planes. Physics of fluids, 11(3), 542-548 (1999) 
45. Pouliquen, Olivier, and François Chevoir.: Dense flows of dry granular material. Comptes Rendus Physique 3.2, 163-175 (2002)

46. Pouliquen, Olivier, and Forterre, Yoel:: A non-local rheology for dense granular flows. Philosophical Transactions of the Royal Society of London A: Mathematical, Physical and Engineering Sciences 367.1909 5091-5107 (2009)

47. Pouliquen, Olivier. \& Forterre, Yoel.: Friction law for dense granular flows: application to the motion of a mass down a rough inclined plane. Journal of fluid mechanics 453, 133-151 (2002)

48. Pouliquen, O., Cassar, C., Jop, P., Forterre, Y., \& Nicolas, M.: Flow of dense granular material: towards simple constitutive laws. Journal of Statistical Mechanics: Theory and Experiment, (07), P07020 (2006)

49. Saingier, G., Deboeuf, S., \& Lagrée, P. Y.: On the front shape of an inertial granular flow down a rough incline. Physics of Fluids, 28(5), 053302 (2016)

50. Sarno, L., Papa, M., Tai, Y., Carravetta, A., \& Martino, R.: A reliable PIV approach for measuring velocity profiles of highly sheared granular flows. Energy, environmental and structural engineering series. (2014)

51. Savage, S. B.: The mechanics of rapid granular flows. In Advances in applied mechanics (Vol. 24, pp. 289-366). Elsevier. (1984)

52. Schaeffer, D.G.: Instability in the evolution equations describing incompressible granular flow. Journal of differential equations 66 (1), 19-50 (1987)

53. Schaefer, M., Bugnion, L., Kern, M., \& Bartelt, P.: Position dependent velocity profiles in granular avalanches. Granular Matter, 12(3), 327-336 (2010) 
54. Shahinpoor, M., and J. S. S. Siah.: New constitutive equations for the rapid flow of granular materials. Journal of Non-Newtonian Fluid Mechanics 9.1, $147-156(1981)$

55. Shahinpoor, M.: New constitutive equations for the rapid flow of granular materials II. Journal of Non-Newtonian Fluid Mechanics 12.1, 31-38 (1983)

56. Shakibaeinia, A., \& Jin, Y. C.: A weakly compressible MPS method for modeling of open-boundary free-surface flow. International journal for numerical methods in fluids, 63(10), 1208-1232 (2010)

57. Shao, S., Lo, E.Y.M..: Incompressible SPH method for simulating Newtonian and non-Newtonian flows with a free surface. Adv. Water Resour. 26, 787$800(2003)$

58. Sheu, Tony W. H., Chenpeng Chiao, and Chinlong Huang.: "Development of a Particle Interaction Kernel Function in MPS Method for Simulating Incompressible Free Surface Flow,” Journal of Applied Mathematics, vol. 2011, Article ID 793653, 16 pages, doi:10.1155/2011/793653 (2011)

59. Shojaaee, Z., Roux, J. N., Chevoir, F., \& Wolf, D. E.: Shear flow of dense granular materials near smooth walls. I. Shear localization and constitutive laws in the boundary region. Physical Review E, 86(1), 011301 (2012)

60. Shukla, Priyanka, and Meheboob Alam.: Nonlinear vorticity-banding instability in granular plane Couette flow: higher-order Landau coefficients, bistability and the bifurcation scenario. Journal of Fluid Mechanics 718, 131$180(2013)$

61. Silbert, Leonardo E., James W. Landry, and Gary S. Grest.: Granular flow down a rough inclined plane: transition between thin and thick piles. Physics of Fluids 15.1, 1-10 (2003) 
62. Stanislav Parez, Einat Aharonov, Renaud Toussaint.: Unsteady granular flows down an inclined plane. Physical Review E, American Physical Society (APS), 93, pp.042902. <10.1103/Phys-RevE.93.042902>. <hal-01340882> (2016)

63. Souto-Iglesias, A., Macià, F., González, L. M., \& Cercos-Pita, J. L.: On the consistency of MPS. Computer Physics Communications, 184(3), 732-745 (2013)

64. Sulsky, Deborah, Zhen Chen, and Howard L. Schreyer.: A particle method for history-dependent materials. Computer methods in applied mechanics and engineering 118.1-2 179-196 (1994)

65. Szewc, Kamil.: Smoothed particle hydrodynamics modeling of granular column collapse. Granular Matter 19.13 (2017)

66. Tankeo, Merline, Patrick Richard, and Édouard Canot.: Analytical solution of the $\mu(\mathrm{I})$-rheology for fully developed granular flows in simple configurations. Granular Matter 15.6 881-891 (2013)

67. Thielicke, W., \& Stamhuis, E.: PIVlab-towards user-friendly, affordable and accurate digital particle image velocimetry in MATLAB. Journal of Open Research Software, 2(1) (2014)

68. Tordesillas, A., Pucilowski, S., Lin, Q., Peters, J. F., \& Behringer, R. P.: Granular vortices: identification, characterization and conditions for the localization of deformation. Journal of the Mechanics and Physics of Solids, 90, 215-241. (2016)

69. Xu, Tibing, and Yee-Chung Jin.: Modeling free-surface flows of granular column collapses using a mesh-free method. Powder Technology 291, 20-34 (2016) 
70. Xu, T., Jin, Y. C., Tai, Y. C., \& Lu, C. H.: Simulation of velocity and shear stress distributions in granular column collapses by a mesh-free method. Journal of Non-Newtonian Fluid Mechanics, 247, 146-164 (2017)

71. Zhang, Y. X. and Wan, D. C.: "Numerical simulation of liquid sloshing in low-filling tank by MPS," Chin. J. Hydrodyn. 27, 100-107 (2012) 